\title{
Mitofusin 1 and 2 regulation of mtDNA content is a critical determinant of glucose homeostasis
}

\author{
Vaibhav Sidarala \\ University of Michigan \\ Jie Zhu \\ University of Michigan \\ Gemma Pearson \\ University of Michigan \\ Emma Reck \\ University of Michigan \\ Brett Kaufman \\ University of Pittsburgh https://orcid.org/0000-0003-4767-4937 \\ Scott Soleimanpour ( $\nabla$ ssol@umich.edu ) \\ University of Michigan https://orcid.org/0000-0001-6777-4498
}

\section{Article}

Keywords: Mitofusin 1 and 2 (Mfn1 and Mfn2), mtDNA content, glucose homeostasis, diabetes

Posted Date: April 15th, 2021

DOI: https://doi.org/10.21203/rs.3.rs-310904/v1

License: (c) (i) This work is licensed under a Creative Commons Attribution 4.0 International License.

Read Full License 
Mitofusin 1 and 2 regulation of mtDNA content is a critical determinant of glucose homeostasis

Vaibhav Sidarala $^{1^{*}}$; Jie Zhu ${ }^{1 *}$; Gemma L. Pearson ${ }^{1}$; Emma C. Reck ${ }^{1}$; Brett A. Kaufman ${ }^{2}$; and Scott A. Soleimanpour ${ }^{1,3}$

${ }^{1}$ Division of Metabolism, Endocrinology \& Diabetes and Department of Internal Medicine, University of Michigan Medical School, Ann Arbor, MI 48105, USA.

${ }^{2}$ Vascular Medicine Institute, Division of Cardiology, Department of Medicine, University of Pittsburgh School of Medicine, Pittsburgh, PA, 15260, USA

${ }^{3}$ VA Ann Arbor Healthcare System, Ann Arbor, MI 48105, USA.

${ }^{*}$ These authors contributed equally to this work

\section{Corresponding Author}

Scott A. Soleimanpour, MD, 1000 Wall Street, Brehm Tower Room 5317, Ann Arbor, MI 48105.

Phone: (734) 763-0528 E-mail: ssol@med.umich.edu 


\section{ABSTRACT}

The dynamin-like GTPases Mitofusin 1 and 2 (Mfn1 and Mfn2) are essential for mitochondrial function, which has been principally attributed to their regulation of fission/fusion dynamics. Here, we report that Mfn1 and 2 are critical for glucose-stimulated insulin secretion (GSIS) primarily through control of mtDNA content. Whereas Mfn1 and Mfn2 individually were dispensable for glucose homeostasis, combined Mfn1/2 deletion in $\beta$-cells reduced mtDNA content, induced mitochondrial fragmentation, and impaired respiratory function, ultimately resulting in severe glucose intolerance. Importantly, gene dosage studies unexpectedly revealed that Mfn $1 / 2$ control of glucose homeostasis was dependent on maintenance of mtDNA content, rather than mitochondrial structure. Indeed, pharmacologic mitofusin agonists rescued islet mtDNA depletion due to mitofusin deficiency independent of changes on mitochondrial structure. Mfn1/2 maintain mtDNA content by regulating the expression of the crucial mitochondrial transcription factor Tfam, as Tfam overexpression ameliorated the reduction in mtDNA content and GSIS in Mfn1/2deficient $\beta$-cells. Thus, the primary physiologic role of Mfn1 and 2 in $\beta$-cells is coupled to preservation of mtDNA content rather than mitochondrial architecture, and Mfn1 and 2 may be promising targets to overcome mitochondrial dysfunction and restore glucose control in diabetes. 


\section{INTRODUCTION}

Mitochondrial dynamics, the balance of fusion and fission of mitochondrial networks, is essential to control mitochondrial structure. Mitochondrial fission and fusion are governed by several key proteins that maintain mitochondrial quality control $(1,2)$. Fission is primarily regulated by the GTPase dynamin-related protein 1 (Drp1), while fusion is controlled by both outer and inner mitochondrial membrane machinery. The dynamin-related large GTPase optic atrophy protein 1 (Opa1) controls inner membrane fusion, while outer membrane fusion is controlled by two GTPases known as Mitofusin 1 and 2 (Mfn1 and Mfn2). Mitofusin 1 and 2 share $\sim 80 \%$ sequence similarity and contain homologous functional domains, suggesting they play both overlapping and unique roles in metabolic function largely ascribed to their control of mitochondrial structure (3).

Imbalances in mitochondrial dynamics in metabolically active tissues have been implicated in many human diseases, including neurodegenerative conditions, cancer, cardiovascular disease, and diabetes (2). Mitochondria provide the energy necessary for $\beta$-cell insulin release $(4,5)$, and abnormalities in mitochondrial structure and bioenergetics have been observed in the $\beta$-cells of humans with type 2 diabetes (T2D; $(6,7)$ ). Thus, strategies to understand and overcome mitochondrial dysfunction in $\beta$-cells are of great appeal for the treatment of diabetes. However, the role of the core fission/fusion machinery in $\beta$-cells is less clear. Reduced $\beta$-cell Mfn2 expression has been observed secondary to the deposition of toxic islet amyloid polypeptide oligomers observed in T2D as well as in mouse models of T2D $(8,9)$, yet the functions of Mfn1 and Mfn2 in $\beta$-cells in vivo are unexplored.

Here we elucidate a key physiologic role for Mfn1 and 2 in the maintenance of glucose homeostasis that unexpectedly occurs through the preservation of $\beta$-cell mtDNA content, rather than mitochondrial structure. Utilizing several genetic mouse models, high resolution imaging, and transcriptomic profiling, we show that Mfn1 and 2 act in tandem to preserve $\beta$-cell mitochondrial health and, consequently, glucose-stimulated insulin secretion (GSIS). Mfn1 and 2 maintain mtDNA content by preventing loss of the mitochondrial transcription factor Tfam, which 
is a master regulator of mtDNA copy number control and mtRNA expression $(10,11)$. Indeed, adenoviral overexpression of Tfam ameliorates reductions in mtDNA content and GSIS in Mfn1/2deficient $\beta$-cells, illustrating the critical importance of mtDNA copy number control by Mfn $1 / 2$ to promote glucose homeostasis. 


\section{RESULTS}

Combined deficiency of Mfn1 and Mfn2 in $\beta$-cells leads to severe glucose intolerance and reduced glucose-stimulated insulin secretion

Mitochondrial health is vital to support $\beta$-cell insulin secretory responses to glucose or other nutrient stimuli. We hypothesized that Mfn1 and Mfn2 are required for GSIS in $\beta$-cells through their regulation of mitochondrial function. To test this hypothesis, we generated mice bearing $\beta$ cell specific deletion of Mfn1 alone (Mfn $1^{\text {loxP/loxP}} ;$ Ins1-Cre), Mfn2 alone (Mfn2 $2^{\text {loxP/loxP }} ;$ Ins1-Cre), and

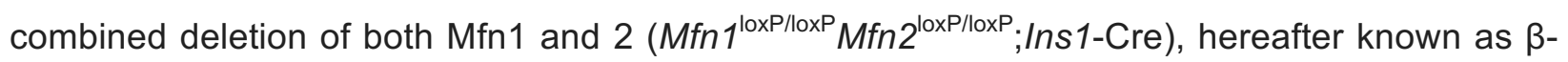
$\operatorname{Mfn} 1^{\mathrm{KO}}, \beta-\mathrm{Mfn} 2^{\mathrm{KO}}$, and $\beta-\mathrm{Mfn} 1 / 2^{\mathrm{DKO}}$ mice, respectively. $\beta-\mathrm{Mfn} 1^{\mathrm{KO}}, \beta-\mathrm{Mfn} 2^{\mathrm{KO}}$, and $\beta-\mathrm{Mfn} 1 / 2^{\mathrm{DKO}}$ mice each exhibited an efficient reduction in Mfn1 and/or Mfn2 islet protein expression, respectively, when compared to littermate controls (Ctrl; Figures 1A-B). We did not observe a compensatory increase in Mfn2 expression in $\beta-M f n 1^{\mathrm{KO}}$ islets, nor in Mfn1 expression in $\beta-M f n 2^{\mathrm{KO}}$ islets (Figures 1A-B). Further, we did not observe differences in expression of the other core fission/fusion proteins, Drp1 and Opa1, following deletion of Mfn1 or Mfn2 (Figure S1A).

While loss of either Mfn1 or Mfn2 alone has been shown to impair glucose homeostasis in other metabolic tissues $(3,12)$, we were surprised to find that both $\beta-\mathrm{Mfn} 1^{\mathrm{KO}}$ and $\beta-\mathrm{Mfn} 2^{\mathrm{KO}}$ mice exhibited normal glucose homeostasis during an intraperitoneal glucose tolerance test (IPGTT; Figure $1 C$ ). We also observed no changes in insulin secretion in single $\beta-M f n 1^{\mathrm{KO}}$ or $\beta-M f n 2^{\mathrm{KO}}$ mice after glucose administration in vivo (Figure 1D) or in isolated islets (Figure 1E). However, combined deletion of both Mfn1 and Mfn2 resulted in severe glucose intolerance (Figure 1B; $p$ $<0.005$ by ANOVA vs. Ctrl), due to markedly reduced GSIS (Figures 1D-E). Reductions in GSIS in $\beta-M f n 1 / 2^{\mathrm{DKO}}$ mice were not secondary to changes in insulin content (Figure S1B).

To evaluate other etiologies of glucose intolerance in $\beta-M f n 1 / 2^{\mathrm{DKO}}$ mice, we first measured $\beta$-cell mass. We observed no significant differences in $\beta$-cell mass between genotypes (Figure 1F). There were also no differences in body weight or peripheral insulin sensitivity between the groups (Figures S1C-D). Taken together, these studies suggest that Mfn1 and 2 are individually 
dispensable for glycemic control and GSIS, but together play vital complementary roles for the maintenance of glucose tolerance and $\beta$-cell insulin release.

\section{Mfn1 and 2 regulate $\beta$-cell mitochondrial structure and respiratory function}

Given the connections between mitochondrial function and dynamics, we asked whether mitochondrial respiration and architecture would be defective in the islets of $\beta-\mathrm{Mfn} 1 / 2^{\mathrm{DKO}}$ mice. Indeed, islets isolated from $\beta-\mathrm{Mfn} 1 / 2^{\mathrm{DKO}}$ mice displayed a reduced glucose-stimulated oxygen consumption rate (OCR; Figure $2 \mathrm{~A}$ ) when compared to Ctrl islets. We did not observe differences in glycolysis as measured by extracellular acidification rate (data not shown). Differences in glucose-stimulated OCR between $\mathrm{Ctrl}$ and $\beta-\mathrm{Mfn} 1 / 2^{\mathrm{DKO}}$ islets were no longer observed following exposure to the Complex III inhibitor antimycin A, suggesting that Mfn1/2-deficient islets primarily possess a defect in $\beta$-cell mitochondrial respiration (Figure $2 \mathrm{~A}$ ).

Loss of key mediators of mitochondrial fusion disrupt mitochondrial network balance and favor increased mitochondrial fission, often leading to the appearance of increased mitochondrial fragmentation (13). We thus examined mitochondrial structure in Mfn1- and/or Mfn2-deficient $\beta$ cells by two independent and complementary methods. First, we assessed $\beta$-cell mitochondrial ultrastructure by transmission electron microscopy (TEM). While $\beta$-cells from all groups had the expected appearance of electron dense insulin granules, $\beta$-cells from $\beta$-Mfn $1 / 2^{\mathrm{DKO}}$ mice had reductions in mitochondrial aspect ratio, form factor, and perimeter, and increased mitochondrial circularity (Figures 2B-C), indicating smaller, fragmented mitochondria. Secondly, we examined mitochondrial morphology and networks utilizing high-resolution deconvolution imaging of the mitochondrial marker succinate dehydrogenase A (SDHA) to generate three-dimensional (3D) reconstructions of $\beta$-cell mitochondria $(14,15)$. Consistent with our findings using TEM, the 3D mitochondrial appearance was similar between $\mathrm{Ctrl}$ and single $\beta-\mathrm{Mfn} 1^{\mathrm{KO}}$ or $\beta-\mathrm{Mfn} 2^{\mathrm{KO}} \beta$-cells (Figure 2D). However, Mfn1/2-double deficient mice showed a dramatic increase in the frequency of smaller, vesiculated $\beta$-cell mitochondrial networks, consistent with fragmentation (Figure 2D). 
3D quantification confirmed that measures of mitochondrial morphology (reduced mitochondrial volume and increased sphericity) and network/branch complexity (reduced branch number and branch junctions) were significantly impaired in $\beta-M f n 1 / 2^{\mathrm{DKO}}$ mice, commensurate with an increase in mitochondrial fragmentation due to decreased mitochondrial fusion (Figure 2E). Together, these data demonstrate that Mfn1 and 2 combine to maintain $\beta$-cell mitochondrial structure and function.

\section{Mfn1 and 2 maintain $\beta$-cell mtDNA content}

Mitochondrial DNA copy number is vital for $\beta$-cell function and glucose homeostasis $(16,17)$. Thus, we next examined whether mtDNA content was altered by loss of Mfn1 and/or Mfn2 in $\beta$ cells. We observed a small, yet significant, reduction in mtDNA content in islets of $\beta-M f n 2^{\mathrm{KO}}$ mice that was not observed in islets of $\beta-M f n 1^{\mathrm{KO}}$ mice (Figure $3 \mathrm{~A}$ ). Interestingly, mtDNA depletion substantially worsened in the islets of $\beta-M f n 1 / 2^{\mathrm{DKO}}$ mice (Figure $3 \mathrm{~A}$ ), suggesting that loss of Mfn 1 exacerbates the depletion of mtDNA caused by Mfn2 deficiency. We next assessed expression of the 13 mitochondrially encoded genes and observed a consistent and significant reduction in mtRNA expression in $\beta-M f n 2^{\mathrm{KO}}$ islets that was exacerbated in $\beta-\mathrm{Mfn} 1 / 2^{\mathrm{DKO}}$ mice (Figure 3B). Concordantly, examination of protein expression of mitochondrial OXPHOS subunits by Western blot also revealed a reduction of the mitochondrially encoded subunit of Complex IV, mt-Co1, in $\beta-\mathrm{Mfn} 2^{\mathrm{KO}}$ islets and $\beta-\mathrm{Mfn} 1 / 2^{\mathrm{DKO}}$ islets (Figures $3 \mathrm{C}-\mathrm{D}$ ). We also observed significant reductions of nuclear encoded subunits of Complex I (Ndufb8) in both $\beta-M f n 2^{\mathrm{KO}}$ and $\beta-\mathrm{Mfn} 1 / 2^{\mathrm{DKO}}$ islets and Complex III (Uqcrc2) in $\beta-M f n 1 / 2^{\mathrm{DKO}}$ islets, but no significant depletion of nuclear encoded Complex II (Sdhb) and Complex V (Atp5a) (Figures 3C-D). These studies reveal that both Mfn1 and 2 (and Mfn2 alone to a lesser degree) are necessary to maintain mtDNA copy number, mtRNA expression, and expression of OXPHOS subunits in $\beta$-cells. 


\section{Defects in $\beta$-cell mitochondrial fusion induce glucose intolerance upon loss of mtDNA}

content

Our observations showing distinct and overlapping contributions of Mfn1 and 2 in the regulation of $\beta$-cell mitochondrial function led us to hypothesize that loss of mitochondrial fusion would lead to glucose intolerance due to mtDNA depletion. However, approaches to clarify the specific contribution of mtDNA depletion to metabolic dysfunction following imbalances in mitochondrial dynamics have been elusive. To this end, we generated animals harboring only a single functional allele of either Mfn1 $\left(\beta-M f n 1^{+/-} \mathrm{Mfn} 2^{\mathrm{KO}}\right)$ or $\mathrm{Mfn} 2\left(\beta-\mathrm{Mfn} 1^{\mathrm{KO}} \mathrm{Mfn} 2^{+/}\right)$to ascertain the relative importance of mtDNA content and structure in the maintenance of glycemic control. For robustness of interpretation, these experiments were performed simultaneously alongside littermate controls and $\beta-M f n 1 / 2^{\mathrm{DKO}}$ mice as previously presented (Figures 1-3). As expected, neither $\beta-M f n 1^{+/-} \mathrm{Mfn} 2^{\mathrm{KO}}$ nor $\beta-M f n 1^{\mathrm{KO}} \mathrm{Mfn} 2^{+/-}$mice developed changes in $\beta$-cell mass, body weight, or insulin sensitivity (Figures S2A-C). Interestingly, 3D quantification of mitochondrial morphology and network integrity revealed that, similar to $\beta-\mathrm{Mfn} 1 / 2^{\mathrm{DKO}}$ mice, a single allele of either Mfn1 or Mfn2 was not sufficient to maintain $\beta$-cell mitochondrial structure and led to mitochondrial fragmentation (Figure 4A-B).

Despite mitochondrial fragmentation in both $\beta-\mathrm{Mfn}^{+/-} \mathrm{Mfn}^{\mathrm{KO}}$ and $\beta-\mathrm{Mfn} 1^{\mathrm{KO}} \mathrm{Mfn}^{+/-}$mice, only $\beta-M f n 1^{+/-} \mathrm{Mfn} 2^{\mathrm{KO}}$ mice developed glucose intolerance and impaired GSIS similar to that of $\beta$ Mfn $1 / 2^{\mathrm{DKO}}$ mice (Figures 4C-D). We also found significant reductions in mtDNA content associated with the loss of GSIS observed in $\beta-\mathrm{Mfn} 1^{+/} \mathrm{Mfn} 2^{\mathrm{KO}}$ and $\beta-\mathrm{Mfn} 1 / 2^{\mathrm{DKO}}$ mice (Figure 4E). Thus, maintenance of a single functional allele of $M f n 2$, but not $M f n 1$, is able to preserve glucose homeostasis, mtDNA content, and insulin secretion, despite being insufficient to maintain $\beta$-cell mitochondrial network integrity. Importantly, these results disjoin $\beta$-cell function from changes in mitochondrial morphology, and implicate mtDNA copy number as the vital purveyor of glycemic control following loss of mitochondrial fusion. 
Mitofusin agonists restore mtDNA content independent of changes in mitochondrial structure

We next asked if pharmacologically targeting mitofusins could restore mtDNA levels in models of mitofusin deficiency independent of changes in mitochondrial structure. To this end, we employed recently described tandem mitofusin agonists, (2-\{2-[(5-cyclopropyl-4-phenyl-4H-1,2,4-triazol-3yl)sulfanyl]propanamido\}-4H,5H,6H-cyclopenta[b]thiophene-3-carboxamide and 1-[2-(benzylsulfanyl)ethyl]-3-(2-methylcyclohexyl)urea (hereafter called Mfn agonists), which together mimic an open Mfn2 conformation favoring mitochondrial fusion but require some residual mitofusin levels to be effective (18). We initially treated control and $\beta-M f n 2^{\mathrm{KO}}$ islets, which exhibit reduced mtDNA content without changes in mitochondrial structure (Figures $3 \mathrm{~A}$ and $2 \mathrm{~B}-\mathrm{E}$ ). To avoid systemic effects of modifying mitochondrial fission/fusion balance in other tissues (1), we utilized Mfn agonists on isolated islets. Interestingly, Mfn agonists restored mtDNA content to baseline in $\beta$ Mfn2 ${ }^{\mathrm{KO}}$ islets (Figure $5 \mathrm{~A}$ ).

We next evaluated the effects of Mfn agonists in other models of mitofusin deficiency, as Mfn2 expression is reduced in the islets of models of T2D (9). We assessed the expression of Mfn1 and 2 in islets isolated from the leptin receptor deficient $d b / d b$ mouse model, which develops obesity, progressive $\beta$-cell mitochondrial and insulin secretory dysfunction, and eventual $\beta$-cell failure (19-23). We observed that Mfn2, but not Mfn1, protein levels were reduced in islets of 1012 week old $d b / d b$ mice when compared to $d b /+$ controls (Figure $5 \mathrm{~B}$ ), measured at an age prior to $\beta$-cell failure $(19,20)$. Similar to observations in $\beta-M f n 2^{\mathrm{KO}}$ islets, $d b / d b$ islets exhibited reduced mtDNA content without alterations in mitochondrial structure (Figures 5C-D). Concordantly, treatment with Mfn agonists also rescued mtDNA levels in $d b / d b$ islets, while leaving mitochondrial structure unchanged (Figures $5 \mathrm{C}-\mathrm{D}$ ). We also observed that Mfn agonists modestly improved GSIS in the islets of $d b / d b$ mice (Figure 5E). Taken together, these results indicate that Mfn agonists restore mtDNA content independent of changes in mitochondrial structure. Moreover, 
these results extend the association between mitofusin deficiency, mtDNA depletion, and impaired GSIS in $\beta$-cells.

\section{Mfn1 and 2 deficiency induces a transcriptional signature consistent with defects in mitochondrial metabolism and alterations in mtDNA replication}

To begin to understand the mechanisms underlying control of mtDNA content by Mfn1 and 2, we analyzed bulk RNA sequencing (RNAseq) data generated from islets of $\beta-M f n 1^{\mathrm{KO}}, \beta-M f n 2^{\mathrm{KO}}$, and $\beta-\mathrm{Mfn} 1 / 2^{\mathrm{DKO}}$ mice as well as littermate controls. Initial hierarchical clustering analyses based on the top 500 most highly expressed genes revealed that only $\beta-\mathrm{Mfn} 1 / 2^{\mathrm{DKO}}$ islets clustered by genotype, while we did not observe discrete clustering across Ctrl or single $\beta$-Mfn $1^{\mathrm{KO}}$ and $\beta$ $\mathrm{Mfn} 2^{\mathrm{KO}}$ islets due to very few differences in expression between these groups (Figures $6 \mathrm{~A}$ and S3A-B). Gene ontology and pathway analyses of differentially expressed genes from $\beta-\mathrm{Mfn} 1 / 2^{\mathrm{DKO}}$ islets revealed significant changes related to endocrine hormone/insulin secretion, $\beta$-cell signature genes, as well as several metabolic pathways (Figures 6B and S3C-D), consistent with the defects in $\beta$-cell function and mitochondrial metabolism observed in $\beta-M f n 1 / 2^{\mathrm{DKO}}$ mice.

The nearly 1,000 significantly dysregulated genes in $\beta-M f n 1 / 2^{\mathrm{DKO}}$ islets included downregulation of mitochondrial encoded transcripts, as well as key $\beta$-cell signature genes such as Glut2 and MafA (Figures $6 \mathrm{C}$ and 6E). We also observed increases in expression of regulators of triglyceride metabolism, including Angptl4 and Angptl8 (24), the glycolytic/gluconeogenic enzyme AldoB, which is highly upregulated in models of $\beta$-cell mitochondrial dysfunction and in T2D islets $(25,26)$, as well as Nrf1, a key regulator of mitochondrial biogenesis (Figures $6 \mathrm{C}, 6 \mathrm{E}$, and S3D; (27)). To resolve additional mitochondrial specific effectors that were differentially regulated following the loss of Mfn1 and 2, we next overlaid our RNAseq data on MitoCarta 2.0 (28), which contains a compendium of targets with strong evidence for localization to the mitochondria (Figure 6D). This revealed increased expression of several genes related to mtDNA replication machinery or associated with the mitochondrial nucleoid (Figures 6D-E), including the 
critical mtDNA topoisomerase Top1mt (29), the mitochondrial co-chaperone Dnaja3/Tid1, which maintains mtDNA integrity (30), and the mitochondrial pyrimidine transporter Slc25a33, which is essential for mtDNA replication (31). We also observed upregulation in the mitochondrial AAA+ protease LonP1, which interacts with and regulates the stability of numerous proteins at the mitochondrial nucleoid (Figures 6D-E; (32-34)). Importantly, we did not observe changes in expression of other fission/fusion genes or regulators of mtRNA transcription, mtDNA repair, sirtuins, or mitophagy (Figure S3D). Taken together, these data indicate that Mfn1/2-deficiency induced a transcriptional signature consistent with an activation of mtDNA replication to potentially compensate for $\beta$-cell mtDNA depletion.

\section{Mfn1 and 2 act through Tfam to regulate $\beta$-cell mtDNA copy number}

The mechanisms underlying regulation of mtDNA by mitochondrial fusion have been controversial, and previous studies implicate several pathways that could reduce mtDNA content, including genome instability/increased mtDNA mutations, enhanced mitophagy, or impaired mtDNA replication (35-37). As our studies were completed in young mice, which bear infrequent mtDNA mutations (38), and we did not observe differences in expression of mtDNA repair enzymes (Figure S3D), we focused on assessments of mitophagy and mtDNA replication to better resolve the significant reduction of mtDNA observed in $\beta-\mathrm{Mfn} 1 / 2^{\mathrm{DKO}}$ islets. We first assessed rates of mitophagy following incubation of control and $\beta-M f n 1 / 2^{\mathrm{DKO}}$ islets with the cell permeable Mtphagy dye, and did not observe differences in mitophagy between genotypes (Figure S3E). We also did not observe a difference in frequency of autophagosomes bearing mitochondria by TEM, or changes in essential genes in the mitophagy pathway between control and $\beta-\mathrm{Mfn} 1 / 2^{\mathrm{DKO}}$ islets (Figure S3D and data not shown).

To assess regulators of $\beta$-cell mtDNA replication, we examined expression of the replisome proteins Ssbp1, Twinkle, and Polg, as well as Tfam, a nucleoid protein which both regulates mtDNA content and whose levels are proportional to mtDNA copy number (10). While 
replisome proteins were largely unchanged, we observed a significant reduction in Tfam protein in $\beta$-Mfn1/2 ${ }^{\mathrm{DKO}}$ islets (Figures 7A-B). Tfam mRNA expression was unchanged in $\beta-\mathrm{Mfn} 1 / 2^{\mathrm{DKO}}$ mice (Figure S3D), suggesting the reduction in Tfam protein occurred post-transcriptionally. We further observed a significant increase in the level of LonP1 protein, which has been shown to lead to Tfam protein turnover $(39,40)$. We additionally confirmed reductions in Tfam and increases in LonP1 protein in $\beta-M f n 1^{+/-} \mathrm{Mfn} 2^{\mathrm{KO}}$ islets (Figure S4), correlating with the mtDNA depletion, glucose intolerance, and impaired GSIS observed in these mice (Figures 4C-E). In contrast, $\beta$ $\mathrm{Mfn}^{\mathrm{KO}} \mathrm{Mfn}^{+/-}$mice, which develop mitochondrial fragmentation without glucose intolerance or mtDNA depletion, did not exhibit changes in Tfam and LonP1 (Figure S4 and Figures 4C-E). These results suggest that changes in Tfam and LonP1 expression following Mfn1/2 deficiency were not due to mitochondrial fragmentation.

To test for defects in mtDNA replication, we next assessed initiation of first strand replication. First strand synthesis in mitochondrial replication begins in the D-loop. However, a single stranded (ss) molecule that forms the classic D-loop structure called 7S, which is a nonreplicative structure, shares this sequence. It is the elongation of the $7 \mathrm{~S}$ sequence into the nearby region (Cytb) that demonstrates committed initiation of replication. Here we assessed the levels of ss and dsDNA at specific sequences by quantitative PCR to overcome expected sample limitations from studies with mouse pancreatic islets. Notably, import of nascent Tfam has been shown to increase the generation of 7S DNA due to its transcription initiation activity (41). We found a trend towards reduced total ss $7 \mathrm{~S}$ sequences normalized to ds mtDNA in $\beta-\mathrm{Mfn} 1 / 2^{\mathrm{DKO}}$ islets (Figure $7 \mathrm{C}$ ). However, the rate of $7 \mathrm{~S}$ sequence extension into committed first strand replication was increased in $\beta-\mathrm{Mfn} 1 / 2^{\mathrm{DKO}}$ islets (Figure $7 \mathrm{C}$ ), which could represent a compensatory response to mtDNA depletion.

Increased first strand replication intermediates heighten the levels of displaced second strand template, which is bound by the single strand binding protein Ssbp1 (42). Consistent with ss qPCR studies, in situ levels of Ssbp1 at the mtDNA nucleoid (detected by perinuclear anti-DNA 
antibody staining), were significantly elevated in Mfn1/2-deficient $\beta$-cells (Figures 7D-E). The increase in Ssbp1 localization to mitochondrial nucleoids could represent binding to a higher quantity of single-stranded mtDNA replication intermediates and could also be consistent with observations of impaired completion of mtDNA replication previously reported following Mfn1/2deficiency $(36,43)$. We also observed Ssbp1 nuclear localization (Figures 7D and F), which has been previously observed following mitochondrial stress (44). Further, we detected an increase in the volume of mtDNA nucleoid structures in Mfn1/2-deficient $\beta$-cells by high-resolution 3D imaging (Figures 7G-H), which are similar to previous reports of Tfam-deficiency but could also represent impaired nucleoid distribution $(36,45,46)$. Taken together, our observations of reduced mtDNA content, mtRNA expression, as well as changes in single-stranded mtDNA products, Ssbp1 localization, and nucleoid size following Mfn1/2-deficiency could be suggestive of a role for Tfam in mediating the effects of Mfn1/2 in $\beta$-cell function.

To test whether increasing mtDNA copy number was sufficient to improve $\beta$-cell function in fusion-deficient islets, we overexpressed Tfam in $\beta-M f n 1 / 2^{\mathrm{DKO}}$ and littermate control islets. We transduced intact islets using adenoviral vectors encoding the human form of TFAM, thus allowing us to track overexpression of TFAM using human-specific TFAM anti-sera (Figures 8A-B and S5A). Transduction efficiency in $\beta$-cells was estimated at $\sim 50 \%$ and was similar in both genotypes (Figure S5A and data not shown). As expected, we did not observe a rescue of mitochondrial structural defects in Mfn1/2-deficient $\beta$-cells by TFAM overexpression (data not shown). Despite limitations in transduction efficiency and expression, TFAM overexpression significantly ameliorated the reductions in both GSIS and mtDNA copy number in $\beta-M f n 1 / 2^{\text {DKO }}$ islets (Figures 8C-D and S5B). Therefore, Mfn 1 and 2 direct $\beta$-cell function, at least in part, through the regulation of Tfam-mediated mtDNA copy number control. 


\section{DISCUSSION}

Here, we identify that Mfn1 and 2 promote mitochondrial fitness to fuel glucose-stimulated insulin release in pancreatic $\beta$-cells and maintain glucose homeostasis. Mfn1 or Mfn2 are individually dispensable for $\beta$-cell function, but together coordinately control mitochondrial respiration, structure, and mtDNA content. Mfn1 and 2 were thought to regulate cellular metabolism principally through the control of mitochondrial architecture. Our single allele mouse models directly demonstrate that Mfn1/2-deficiency is coupled to glucose intolerance through mtDNA loss rather than mitochondrial structure. Further, we observe that increased mtDNA copy number following Tfam overexpression is capable of ameliorating impaired GSIS in fusion-deficient $\beta$-cells. Thus, our studies position the maintenance of mtDNA content as the principal task necessary for Mfn1 and 2 to regulate $\beta$-cell function.

Our description of a central role for Mfn1 and 2 in $\beta$-cells through mtDNA content rather than mitochondrial structure elucidates a previously unappreciated role for mitochondrial fusion on cellular metabolic function. Mfn1 and 2 act in a complementary manner to support mitochondrial metabolism from development to adulthood. Both germline and cardiomyocytespecific deletion of Mfn1 and 2 are associated with developmental or early postnatal demise (47, 48), presumed to be primarily caused by defects in mitochondrial network dynamics. Later descriptions of reduced mtDNA levels in constitutive or inducible loss of Mfn1/2 in skeletal muscle did not discern the physiologic importance of mtDNA depletion versus structure following fusiondeficiency $(35,49)$. Our results clarify a primary physiologic impact of mtDNA depletion rather than mitochondrial structure following Mfn1/2 deficiency in $\beta$-cells that may also underlie the physiologic importance of Mfn1 and 2 in other metabolic tissues. To our knowledge, this is the first time that these functions of Mfn1/2 have been separated in a physiological context.

While our data suggest that Tfam mediates the effects of Mfn1/2 on mtDNA content and GSIS in $\beta$-cells, the mechanisms for post-transcriptional loss of Tfam in $\beta-M f n 1 / 2^{\mathrm{DKO}}$ islets are unclear. Loss of Tfam may be due to increased LonP1 expression, which is known to degrade 
Tfam $(39,40)$. Increases in LonP1 expression have also been reported to lead to the instability of nuclear-encoded subunits of Complex I and III, similar to our observations following Mfn1/2deficiency (Figure 3D; (50)), which could further contribute to mitochondrial dysfunction. However, the function and transcriptional regulation of LonP1 in $\beta$-cells are unknown and require future study.

We observe that $\beta-M f n 1 / 2^{\text {DKO }}$ islets attempt unsuccessfully to compensate for mtDNA depletion. Our analyses of first strand replication suggest that Mfn1/2-deficient $\beta$-cells increase the activation of early steps in mtDNA replication, which is supported by increased Ssbp1 localization to nucleoids to potentially bind newly formed ss-mtDNA. Despite these changes, mtDNA content remains low in Mfn1/2-deficient $\beta$-cells, suggesting that the increased commitment to replication is insufficient. Indeed, increased nucleoid Ssbp1 may be engaged in binding abnormal single-stranded replication products due to fusion and replication defects (36, 43). The increased expression of Top1 $\mathrm{mt}$ found in Mfn1/2-deficient $\beta$-cells could also represent a response to increased mitochondrial replication stress (51). As Mfn2 is frequently observed at mitochondrial-ER contacts (MERCs; (52)), which are the sites of mtDNA replication (53), alterations in MERCs in $\beta-M f n 1 / 2^{\mathrm{DKO}}$ islets could possibly lead to impaired mtDNA replication. However, a recent report found that inducible loss of Mfn1/2 in adult $\beta$-cells did not alter MERCdependent ER calcium release (54), and we did not observe ER dilation or overt changes in mitochondrial-ER proximity on transmission EM in $\beta-M f n 1 / 2^{\mathrm{DKO}}$ islets (Fig. $2 \mathrm{~B}$ and data not shown), suggesting Mfn1/2 do not substantially affect $\beta$-cell MERCs. Additional experimentation will be required to dissect the molecular details underlying the connections between mitochondrial fusion and control of $\beta$-cell mtDNA replication.

Mitochondrial fission/fusion disturbances may lead to downstream extramitochondrial effects that could contribute to $\beta$-cell failure, but these have not yet been explored. Notably, $\beta$-cell Drp1 deficiency reduces second phase insulin release and the amplification pathway of insulin secretion (55), and $\beta$-cell Opa1 deficiency impairs entry into the cell cycle, thereby reducing $\beta$ - 
cell replication (56). We detect an increase of nuclear Ssbp1 following loss of mitochondrial fusion, which has been suggested to activate a mitochondrial stress response (44). We further observe significant hyperglycemia, reduced Tfam expression, and mtDNA depletion in young $\beta-M f n 1 / 2^{\text {DKO }}$ mice that could potentially promote eventual glucotoxicity or an age-related loss of $\beta$-cell mass, similar to a previous report of selective Tfam deficiency in $\beta$-cells (17). Indeed, an independent group found that, similar to the GSIS defects we observe following constitutive loss of Mfn1/2 in $\beta$-cells, inducible Mfn1/2-deficiency in adult $\beta$-cells also impairs GSIS, leading to an eventual decline of $\beta$-cell mass with age (54). Investigation of the extramitochondrial effects which emanate from mtDNA depletion and disruptions in mitochondrial networking will be essential to understand how the early defects in mitochondria-potentiated insulin release cause progressive loss of $\beta$-cell mass.

Mitochondrial structural defects and age-related mtDNA depletion combine to impair $\beta$ cell function to increase T2D risk $(6,7,57-59)$, thus, improving mitochondrial function would have significant translational benefits to restore $\beta$-cell health in diabetes. Our studies demonstrate the feasibility of targeting mtDNA copy number to improve GSIS in the setting of mitofusin deficiency. Further, recently identified compounds targeting pyrimidine metabolism to induce Mfn $1 / 2$ transcription (60) will be of interest to determine if they can improve $\beta$-cell function and mtDNA content. Our observation of the upregulation of mitochondrial pyrimidine transporter Slc25a33 in $\beta-\mathrm{Mfn} 1 / 2^{\mathrm{DKO}}$ islets is consistent with links between pyrimidine metabolism and mitochondrial fusion, which suggest these compounds could hold promise to enhance $\beta$-cell mitochondrial function. Future studies will be required to fully characterize the efficacy of overcoming mitochondrial defects in vivo to treat diabetes. 


\section{METHODS}

\section{Animals}

$M f n 1^{\text {loxP/loxP }}$ and Mfn $2^{\text {loxP/loxP }}$ mice possessing loxP sites flanking exon 4 of the Mfn 1 gene and exon 6 of the Mfn2 gene, respectively, were purchased from Jackson Laboratories (61). Mfn $1^{\text {loxP }}$ and Mfn2 ${ }^{\text {loxP }}$ mice were mated to Ins1-Cre (62) to generate experimental groups. Ins1-Cre-alone, Mfn $1^{\text {loxP/loxP }}$ and Mfn $2^{\text {loxP/loxP }}$ mice were phenotypically indistinguishable from each other and combined as controls (Ctrl). Ins1-Cre-alone were also phenotypically indistinguishable from wildtype C57BL/6N controls, consistent with previous reports from our group and others (data not shown; $(14,62))$. All animals were all maintained on the C57BL/6N background. $d b /+$ and $d b / d b$ mice, maintained on the BKS background, were purchased from Jackson Laboratories. Animals were housed on a standard 12-hour light/12-hour dark cycle with ad libitum access to food and water. All studies and endpoints in all figures were completed using both male and female mice, and results from both sexes were combined in all experimental groups.

\section{Mitochondrial respirometry}

Islet respirometry was measured using an XF96 extracellular flux analyzer (Seahorse Bioscience) according to manufacturer's instructions. Briefly, 20 similarly sized islets were plated per well of a Seahorse spheroid plate pre-treated with CellTak (Corning), similar to previously published approaches (63). Prior to respirometry profiling, islets were incubated for 1 hour in an atmospheric $\mathrm{CO}_{2}$ incubator at $37^{\circ} \mathrm{C}$ and supplemented with mouse islet culture media $(64,65)$ comprised of pH 7.4 unbuffered RPMI1640 media (Seahorse Bioscience) and 2mM glucose prior to flux analyzer assays. Analysis was performed at baseline, upon exposure to $20 \mathrm{mM}$ glucose, and again after treatment with $2 \mu \mathrm{M}$ antimycin $\mathrm{A}$. OCR measurements were normalized to islet number confirmed by light microscopy (Zeiss Stemi 2000) after the completion of respirometry assays.

\section{Transmission electron microscopy}

Mouse islets were pelleted, fixed in $3 \%$ glutaraldehyde and $3 \%$ paraformaldehyde in $0.1 \mathrm{M}$ Cacodylate buffer ( $\mathrm{CB} ; \mathrm{pH} 7.2)$ overnight at $4^{\circ} \mathrm{C}$. Islets were then embedded in $2 \%$ agarose as 
described previously (66). Samples were then subjected to osmification in $1.5 \% \mathrm{~K}_{4} \mathrm{Fe}(\mathrm{CN})_{6}+2 \%$ $\mathrm{OsO}_{4}$ in $0.1 \mathrm{CB}$ for $1 \mathrm{~h}$, dehydrated by serial washes in $\mathrm{EtOH}(30 \%, 50 \%, 70 \%, 80 \%, 90 \%, 95 \%$ and $100 \%$ ) and embedded in Spurr's resin by polymerization at $60^{\circ} \mathrm{C}$ for $24 \mathrm{~h}$. Polymerized resins were then sectioned at 90nm thickness using a Leica EM UC7 ultramicrotome and imaged at 70kV using a JEOL 1400 TEM equipped with an AMT CMOS imaging system. Mitochondrial structures (Aspect Ratio, Perimeter, Circularity) were analyzed and quantified by ImageJ. Form factor assessments were analyzed by ImageJ and quantified as described (15).

\section{Mitochondrial morphology and subcellular localization}

Mitochondrial morphology and subcellular localization analyses were performed on immunostained paraffin-embedded mouse pancreas tissue sections and dissociated islet cells as previously described (14). Z-stack images of immunostained pancreatic sections were captured with an IX81 microscope (Olympus) using an ORCA Flash4 CMOS digital camera (Hamamatsu) and subjected to deconvolution (CellSens; Olympus). Mitochondrial morphology and nucleoids was visualized using 3D-renderings generated with Imaris ${ }^{\circledR}$ imaging software (Bitplane). Quantitative 3D assessments of mitochondrial morphology and network were performed on ImageJ using MitoAnalyzer plugin (15). Co-localization analyses were performed on Z-stack images of immunostained dissociated islet cells using the Coloc2 plugin on ImageJ. Changes in relative nucleoid size were quantified from $3 D$ deconvolution images of immunostained dissociated islet cells, detected by perinuclear anti-DNA antibody staining, using ImageJ.

\section{mtDNA content and replication assays}

Relative mtDNA content by qPCR with SYBR-based detection (Universal SYBR Green Supermix; Biorad) was conducted as previously described (67) following DNA isolation with the Blood/Tissue DNeasy kit (Qiagen) according to manufacturer's protocols. First strand mtDNA replication levels were measured by qPCR following digestion by Mnl I, which site specifically digest double stranded DNA (68). Briefly, primers specific to 75 mtDNA (5'АСтСтTCTCTTCCATATGACTATCCC-3' and 5'-GgCCCTGAAGTAAGAACCAGATGT-3'), 
whose amplicon contains a $\mathrm{Mnl}$ I site, were used to quantify relative single-stranded mtDNA forming the D-loop. Single-stranded DNA generated from the extension of $7 \mathrm{~S}$ region was measured using primers specific to CYTB (5'-TGCATACGCCATTCTACGCTCA-3' and 5'GTGATTGGGCGGAATATTAGGCTTC-3'), which also contains an Mnl I site. Primers specific to COXI (5'-GCAGGAGCATCAGTAGACCTAACA-3' and 5'-GCGGCTAGCACTGGTAGTGATAAT3'), whose amplicon does not contain an Mnl I site, were used to quantify non-replicating double stranded mtDNA. The ratio of $7 \mathrm{~S}$ or CYTB amplicons to the COXI amplicon were then calculated after qPCR of Mnl I digested DNA to determine the relative amount of single stranded mtDNA at the D-loop strand or initiation of first strand replication, respectively.

\section{RNA sequencing, differential expression, and functional analysis}

Sequencing was performed by the Advanced Genomics Core at University of Michigan Medical School. Total RNA was isolated and DNase treated using commercially available kits (Omega Biotek and Ambion, respectively). Libraries were constructed and subsequently subjected to $151 \mathrm{bp}$ paired-end cycles on the NovaSeq-6000 platform (Illumina). FastQC (v0.11.8) was used to ensure the quality of data. Reads were mapped to the reference genome GRCm38 (ENSEMBL), using STAR (v2.6.1b) and assigned count estimates to genes with RSEM (v1.3.1). Alignment options followed ENCODE standards for RNA-seq. FastQC was used in an additional post-alignment step to ensure that only high-quality data were used for expression quantitation and differential expression. Data were pre-filtered to remove genes with 0 counts in all samples. Differential gene expression analysis was performed using DESeq2, using a negative binomial generalized linear model (thresholds: linear fold change $>1.5$ or $<-1.5$, Benjamini-Hochberg FDR $\left.\left(P_{\text {adj }}\right)<0.05\right)$. Plots were generated using variations of DESeq2 plotting functions and other packages with R version 3.3.3. Genes were annotated with NCBI Entrez GenelDs and text descriptions. Functional analysis, including candidate pathways activated or inhibited in comparison(s) and GO-term enrichments, was performed using iPathway Guide (Advaita). Primary data deposition at GEO is currently in process. 


\section{Adenoviral transfection}

Isolated mouse islets were distended with Accutase for $1 \mathrm{~min}$ at $37^{\circ} \mathrm{C}$ as described previously (69). Islets were then transduced with $0.15 \mathrm{MOI}$ of Ad.EV (expressing an empty vector) or Ad.hTFAM (expressing human-specific TFAM) for $48 \mathrm{hrs}$ (viral particles purchased from Vector Biolabs). $\beta$-cell transduction was evaluated on dissociated islet cells by immunostaining with $\mathrm{Pdx} 1$ and human-specific TFAM anti-sera.

\section{Flow cytometry}

Following isolation and culture, live mouse islets were exposed to $100 \mathrm{nM}$ Mtphagy dye (Dojindo Molecular Technologies) for 3 hours to assess time-dependent accumulation of mitochondria to acidic organelles by the relative fluorescence intensity of the dye per cell as described $(70,71)$. Islets were then dissociated into single cells, stained with DAPI (Thermo Fisher Scientific) and Fluozin-3 (Thermo Fisher Scientific), and resuspended in phenol red-free islet culture medium as previously described (14). Samples were analyzed on an LSR Fortessa flow cytometer (BD Biosciences). Single cells were gated using forward scatter and side scatter (FSC and SSC, respectively) plots, DAPI staining was used to exclude dead cells, and Fluozin-3 was used to identify $\beta$-cells as previously described $(14,72)$. Mtphagy measurements in $\beta$-cells were made using $488 \mathrm{~nm}$ excitation laser with a $710 \mathrm{~nm}$ emission filter and analyzed using FlowJo (Tree Star Inc.). A total of 5,000 $\beta$-cells was quantified from each independent islet preparation.

\section{Glucose and insulin measurements}

IPGTT, ITT and serum insulin measurements were performed as described previously $(64,65)$. Static incubation assays were performed on isolated mouse islets for insulin release as previously described $(64,65)$.

\section{Islet isolation and cell culture}

Mouse islets were isolated and cultured as previously described $(64,65)$. Cell treatments included DMSO (Fisher) and antimycin A (Sigma). Mitofusin agonist treatment included an equimolar mixture of two previously described compounds $(0.5 \mu \mathrm{M}$ each; (18)), which were administered for 
24 hours (2-\{2-[(5-cyclopropyl-4-phenyl-4H-1,2,4-triazol-3-yl)sulfanyl]propanamido\}-4H,5H,6Hcyclopenta[b]thiophene-3-carboxamide, CAS\# 920868-45-7 and 1-[2-(benzylsulfanyl)ethyl]-3-(2methylcyclohexyl)urea, CAS\# 1007818-44-1; Enamine Ltd.).

\section{Western blotting, quantitative PCR, and immunostaining}

All assays were performed as previously described $(14,64,65)$. $\beta$-cell mass was quantified as previously described (65). Antibodies used for Western blotting and immunostaining are listed in Supplementary Table 1.

\section{Statistics}

In all figures, data are presented as means \pm SEM, and error bars denote SEM. Statistical comparisons were performed using unpaired two-tailed Student's t-tests or one-way ANOVA, followed by Tukey's post-hoc test for multiple comparisons, as appropriate (Prism GraphPad). A $P$ value $<0.05$ was considered significant

\section{Study approval}

Animal studies were approved by the University of Michigan Institutional Animal Care and Use Committee.

\section{Author Contributions}

V.S. conceived, designed and performed experiments, interpreted results, drafted and reviewed the manuscript. J.Z. conceived, designed and performed experiments, interpreted results, and reviewed the manuscript. G.L.P. and E.C.R. designed and performed experiments and interpreted results. B.A.K. designed studies, interpreted results and reviewed the manuscript. S.A.S. conceived and designed the studies, interpreted results, drafted, edited, and reviewed the manuscript. 


\section{Acknowledgements}

S.A.S was supported by the JDRF (CDA-2016-189, SRA-2018-539, COE-2019-861), the NIH (R01 DK108921, U01 DK127747), the Department of Veterans Affairs (I01 BX004444), the Brehm family, and the Anthony family. G.L.P. was supported by the American Diabetes Association (19PDF-063). B.A.K. was supported by the Department of Veterans Affairs (I01 BX004444). The JDRF Career Development Award to S.A.S. is partly supported by the Danish Diabetes Academy and the Novo Nordisk Foundation. We acknowledge the Microscopy, Imaging and Cellular Physiology Core of the University of Michigan DRC (P30 DK020572) for assistance with imaging studies. We thank the University of Michigan Flow Cytometry Core for assistance with flow cytometry studies. Next generation sequencing was carried out in the Advanced Genomics Core at the University of Michigan. We acknowledge support from the Bioinformatics Core of the University of Michigan's Biomedical Research Core Facilities. We thank Drs. K. Claiborn, E. Walker, C. Rutledge, N. Desai, and members of the Soleimanpour laboratory for helpful advice.

\section{Conflict of interest statement}

The authors have declared that no conflict of interest exists. 


\section{REFERENCES}

1. Sebastian D, Palacin M, and Zorzano A. Mitochondrial Dynamics: Coupling Mitochondrial Fitness with Healthy Aging. Trends Mol Med. 2017;23(3):201-15.

2. Westermann B. Mitochondrial fusion and fission in cell life and death. Nat Rev Mol Cell Biol. 2010;11(12):872-84.

3. Schrepfer E, and Scorrano L. Mitofusins, from Mitochondria to Metabolism. Mol Cell. 2016;61(5):683-94.

4. Kaufman BA, Li C, and Soleimanpour SA. Mitochondrial regulation of beta-cell function: maintaining the momentum for insulin release. Molecular aspects of medicine. 2015;42:91-104.

5. Maechler P. Mitochondrial function and insulin secretion. Mol Cell Endocrinol. 2013;379(12):12-8.

6. Anello M, Lupi R, Spampinato D, Piro S, Masini M, Boggi U, et al. Functional and morphological alterations of mitochondria in pancreatic beta cells from type 2 diabetic patients. Diabetologia. 2005;48(2):282-9.

7. Masini M, Martino L, Marselli L, Bugliani M, Boggi U, Filipponi F, et al. Ultrastructural alterations of pancreatic beta cells in human diabetes mellitus. Diabetes Metab Res Rev. 2017;33(6).

8. Safia Costes TG, Alexandra E Butler, Sam Sereda, Orian Shirihai and Peter Cawood Butler. In: Society E ed. The Endocrine Society Annual Meeting. San Diego; 2015.

9. Stoehr JP, Nadler ST, Schueler KL, Rabaglia ME, Yandell BS, Metz SA, et al. Genetic obesity unmasks nonlinear interactions between murine type 2 diabetes susceptibility loci. Diabetes. 2000;49(11):1946-54.

10. Campbell CT, Kolesar JE, and Kaufman BA. Mitochondrial transcription factor A regulates mitochondrial transcription initiation, DNA packaging, and genome copy number. Biochim Biophys Acta. 2012;1819(9-10):921-9.

11. Filograna R, Mennuni M, Alsina D, and Larsson NG. Mitochondrial DNA copy number in human disease: the more the better? FEBS Lett. 2020.

12. Liesa $M$, and Shirihai OS. Mitochondrial dynamics in the regulation of nutrient utilization and energy expenditure. Cell Metab. 2013;17(4):491-506.

13. Twig G, Elorza A, Molina AJ, Mohamed H, Wikstrom JD, Walzer G, et al. Fission and selective fusion govern mitochondrial segregation and elimination by autophagy. The EMBO journal. 2008;27(2):433-46.

14. Sidarala V, Pearson GL, Parekh VS, Thompson B, Christen L, Gingerich MA, et al. Mitophagy protects beta cells from inflammatory damage in diabetes. $\mathrm{JCl}$ Insight. 2020;5(24).

15. Chaudhry A, Shi R, and Luciani DS. A pipeline for multidimensional confocal analysis of mitochondrial morphology, function, and dynamics in pancreatic beta-cells. Am J Physiol Endocrinol Metab. 2020;318(2):E87-E101.

16. Mulder $\mathrm{H}$. Transcribing beta-cell mitochondria in health and disease. Molecular metabolism. 2017;6(9):1040-51.

17. Silva JP, Kohler M, Graff C, Oldfors A, Magnuson MA, Berggren PO, et al. Impaired insulin secretion and beta-cell loss in tissue-specific knockout mice with mitochondrial diabetes. Nature genetics. 2000;26(3):336-40.

18. Rocha AG, Franco A, Krezel AM, Rumsey JM, Alberti JM, Knight WC, et al. MFN2 agonists reverse mitochondrial defects in preclinical models of Charcot-Marie-Tooth disease type 2A. Science. 2018;360(6386):336-41.

19. Guo S, Dai C, Guo M, Taylor B, Harmon JS, Sander M, et al. Inactivation of specific beta cell transcription factors in type 2 diabetes. J Clin Invest. 2013;123(8):3305-16. 
20. Ishida E, Kim-Muller JY, and Accili D. Pair Feeding, but Not Insulin, Phloridzin, or Rosiglitazone Treatment, Curtails Markers of beta-Cell Dedifferentiation in $\mathrm{db} / \mathrm{db}$ Mice. Diabetes. 2017;66(8):2092-101.

21. Li J, Du H, Zhang M, Zhang Z, Teng F, Zhao $Y$, et al. Amorphous solid dispersion of Berberine mitigates apoptosis via iPLA2beta/Cardiolipin/Opa1 pathway in $\mathrm{db} / \mathrm{db}$ mice and in Palmitate-treated MIN6 beta-cells. Int J Biol Sci. 2019;15(7):1533-45.

22. Liu J, Chen Z, Zhang Y, Zhang M, Zhu X, Fan Y, et al. Rhein protects pancreatic betacells from dynamin-related protein-1-mediated mitochondrial fission and cell apoptosis under hyperglycemia. Diabetes. 2013;62(11):3927-35.

23. Zhao Z, Zhang X, Zhao C, Choi J, Shi J, Song K, et al. Protection of pancreatic beta-cells by group VIA phospholipase A(2)-mediated repair of mitochondrial membrane peroxidation. Endocrinology. 2010;151(7):3038-48.

24. Kersten S. Physiological regulation of lipoprotein lipase. Biochim Biophys Acta. 2014;1841(7):919-33.

25. Haythorne E, Rohm M, van de Bunt M, Brereton MF, Tarasov Al, Blacker TS, et al. Diabetes causes marked inhibition of mitochondrial metabolism in pancreatic beta-cells. Nat Commun. 2019;10(1):2474.

26. Gerst F, Jaghutriz BA, Staiger H, Schulte AM, Lorza-Gil E, Kaiser G, et al. The Expression of Aldolase B in Islets Is Negatively Associated With Insulin Secretion in Humans. J Clin Endocrinol Metab. 2018;103(12):4373-83.

27. Scarpulla RC. Transcriptional paradigms in mammalian mitochondrial biogenesis and function. Physiol Rev. 2008;88(2):611-38.

28. Calvo SE, Clauser KR, and Mootha VK. MitoCarta2.0: an updated inventory of mammalian mitochondrial proteins. Nucleic Acids Res. 2016;44(D1):D1251-7.

29. Zhang H, Barcelo JM, Lee B, Kohlhagen G, Zimonjic DB, Popescu NC, et al. Human mitochondrial topoisomerase I. Proc Natl Acad Sci U S A. 2001;98(19):10608-13.

30. $\mathrm{Ng} \mathrm{AC}$, Baird SD, and Screaton RA. Essential role of TID1 in maintaining mitochondrial membrane potential homogeneity and mitochondrial DNA integrity. Mol Cell Biol. 2014;34(8):1427-37.

31. Di Noia MA, Todisco S, Cirigliano A, Rinaldi T, Agrimi G, lacobazzi V, et al. The human SLC25A33 and SLC25A36 genes of solute carrier family 25 encode two mitochondrial pyrimidine nucleotide transporters. J Biol Chem. 2014;289(48):33137-48.

32. Zurita Rendon O, and Shoubridge EA. LONP1 Is Required for Maturation of a Subset of Mitochondrial Proteins, and Its Loss Elicits an Integrated Stress Response. Mol Cell Biol. 2018;38(20).

33. Venkatesh S, Lee J, Singh K, Lee I, and Suzuki CK. Multitasking in the mitochondrion by the ATP-dependent Lon protease. Biochim Biophys Acta. 2012;1823(1):56-66.

34. Lu B, Yadav S, Shah PG, Liu T, Tian B, Pukszta S, et al. Roles for the human ATPdependent Lon protease in mitochondrial DNA maintenance. $J$ Biol Chem. 2007;282(24):17363-74.

35. Chen H, Vermulst M, Wang YE, Chomyn A, Prolla TA, McCaffery JM, et al. Mitochondrial fusion is required for mtDNA stability in skeletal muscle and tolerance of mtDNA mutations. Cell. 2010;141(2):280-9.

36. Silva Ramos E, Motori E, Bruser C, Kuhl I, Yeroslaviz A, Ruzzenente B, et al. Mitochondrial fusion is required for regulation of mitochondrial DNA replication. PLoS Genet. 2019;15(6):e1008085.

37. Sebastian D, Sorianello E, Segales J, Irazoki A, Ruiz-Bonilla V, Sala D, et al. Mfn2 deficiency links age-related sarcopenia and impaired autophagy to activation of an adaptive mitophagy pathway. The EMBO journal. 2016;35(15):1677-93.

38. Ma H, Lee Y, Hayama T, Van Dyken C, Marti-Gutierrez N, Li Y, et al. Germline and somatic mtDNA mutations in mouse aging. PLoS One. 2018;13(7):e0201304. 
39. Matsushima Y, Goto Y, and Kaguni LS. Mitochondrial Lon protease regulates mitochondrial DNA copy number and transcription by selective degradation of mitochondrial transcription factor A (TFAM). Proc Natl Acad Sci $U S A$. 2010;107(43):18410-5.

40. Lu B, Lee J, Nie X, Li M, Morozov YI, Venkatesh S, et al. Phosphorylation of human TFAM in mitochondria impairs DNA binding and promotes degradation by the AAA+ Lon protease. Mol Cell. 2013;49(1):121-32.

41. Gensler S, Weber K, Schmitt WE, Perez-Martos A, Enriquez JA, Montoya J, et al. Mechanism of mammalian mitochondrial DNA replication: import of mitochondrial transcription factor A into isolated mitochondria stimulates 7S DNA synthesis. Nucleic Acids Res. 2001;29(17):3657-63.

42. Pohjoismaki JL, and Goffart S. Of circles, forks and humanity: Topological organisation and replication of mammalian mitochondrial DNA. Bioessays. 2011;33(4):290-9.

43. Gustafson MA, Sullivan ED, and Copeland WC. Consequences of compromised mitochondrial genome integrity. DNA Repair (Amst). 2020;93:102916.

44. Tan K, Fujimoto M, Takii R, Takaki E, Hayashida N, and Nakai A. Mitochondrial SSBP1 protects cells from proteotoxic stresses by potentiating stress-induced HSF1 transcriptional activity. Nat Commun. 2015;6:6580.

45. Kasashima $\mathrm{K}$, Sumitani $\mathrm{M}$, and Endo $\mathrm{H}$. Human mitochondrial transcription factor $\mathrm{A}$ is required for the segregation of mitochondrial DNA in cultured cells. Exp Cell Res. 2011;317(2):210-20.

46. West AP, Khoury-Hanold W, Staron M, Tal MC, Pineda CM, Lang SM, et al. Mitochondrial DNA stress primes the antiviral innate immune response. Nature. 2015;520(7548):553-7.

47. Chen H, Detmer SA, Ewald AJ, Griffin EE, Fraser SE, and Chan DC. Mitofusins Mfn1 and Mfn2 coordinately regulate mitochondrial fusion and are essential for embryonic development. J Cell Biol. 2003;160(2):189-200.

48. Papanicolaou KN, Kikuchi R, Ngoh GA, Coughlan KA, Dominguez I, Stanley WC, et al. Mitofusins 1 and 2 are essential for postnatal metabolic remodeling in heart. Circ Res. 2012;111(8):1012-26.

49. Bell MB, Bush Z, McGinnis GR, and Rowe GC. Adult skeletal muscle deletion of Mitofusin 1 and 2 impedes exercise performance and training capacity. J Appl Physiol (1985). 2019;126(2):341-53.

50. Peter B, Waddington CL, Olahova M, Sommerville EW, Hopton S, Pyle A, et al. Defective mitochondrial protease LonP1 can cause classical mitochondrial disease. Hum Mol Genet. 2018;27(10):1743-53.

51. Pommier Y, Sun Y, Huang SN, and Nitiss JL. Roles of eukaryotic topoisomerases in transcription, replication and genomic stability. Nat Rev Mol Cell Biol. 2016;17(11):70321.

52. Sebastian D, Hernandez-Alvarez MI, Segales J, Sorianello E, Munoz JP, Sala D, et al. Mitofusin 2 (Mfn2) links mitochondrial and endoplasmic reticulum function with insulin signaling and is essential for normal glucose homeostasis. Proc Natl Acad Sci U S A. 2012;109(14):5523-8.

53. Lewis SC, Uchiyama LF, and Nunnari J. ER-mitochondria contacts couple mtDNA synthesis with mitochondrial division in human cells. Science. 2016;353(6296):aaf5549.

54. Georgiadou E, Muralidharan C, Martinez M, Chabosseau P, Tomas A, Wern FYS, et al. Mitofusins <em $>$ Mfn $1</ e m>$ and $<$ em $>M f n 2</ e m>$ are required in the $\beta$-cell to preserve mitochondrial architecture and insulin secretion. bioRxiv. 2021:2020.04.22.055384.

55. Hennings TG, Chopra DG, DeLeon ER, VanDeusen HR, Sesaki H, Merrins MJ, et al. In Vivo Deletion of beta-Cell Drp1 Impairs Insulin Secretion Without Affecting Islet Oxygen Consumption. Endocrinology. 2018;159(9):3245-56. 
56. Zhang Z, Wakabayashi N, Wakabayashi J, Tamura Y, Song WJ, Sereda S, et al. The dynamin-related GTPase Opa1 is required for glucose-stimulated ATP production in pancreatic beta cells. Mol Biol Cell. 2011;22(13):2235-45.

57. Nile DL, Brown AE, Kumaheri MA, Blair HR, Heggie A, Miwa S, et al. Age-related mitochondrial DNA depletion and the impact on pancreatic Beta cell function. PLoS One. 2014;9(12):e115433.

58. Cree LM, Patel SK, Pyle A, Lynn S, Turnbull DM, Chinnery PF, et al. Age-related decline in mitochondrial DNA copy number in isolated human pancreatic islets. Diabetologia. 2008;51(8):1440-3.

59. Chinnery PF, Mowbray C, Patel SK, Elson JL, Sampson M, Hitman GA, et al. Mitochondrial DNA haplogroups and type 2 diabetes: a study of 897 cases and 1010 controls. J Med Genet. 2007;44(6):e80.

60. Miret-Casals L, Sebastian D, Brea J, Rico-Leo EM, Palacin M, Fernandez-Salguero PM, et al. Identification of New Activators of Mitochondrial Fusion Reveals a Link between Mitochondrial Morphology and Pyrimidine Metabolism. Cell Chem Biol. 2018;25(3):26878 e4.

61. Chen H, McCaffery JM, and Chan DC. Mitochondrial fusion protects against neurodegeneration in the cerebellum. Cell. 2007;130(3):548-62.

62. Thorens B, Tarussio D, Maestro MA, Rovira M, Heikkila E, and Ferrer J. Ins1(Cre) knockin mice for beta cell-specific gene recombination. Diabetologia. 2015;58(3):558-65.

63. Taddeo EP, Stiles L, Sereda S, Ritou E, Wolf DM, Abdullah M, et al. Individual islet respirometry reveals functional diversity within the islet population of mice and human donors. Molecular metabolism. 2018;16:150-9.

64. Pearson G, Chai B, Vozheiko T, Liu X, Kandarpa M, Piper RC, et al. Clec16a, Nrdp1, and USP8 Form a Ubiquitin-Dependent Tripartite Complex That Regulates beta-Cell Mitophagy. Diabetes. 2018;67(2):265-77.

65. Soleimanpour SA, Gupta A, Bakay M, Ferrari AM, Groff DN, Fadista J, et al. The diabetes susceptibility gene Clec16a regulates mitophagy. Cell. 2014;157(7):1577-90.

66. Soleimanpour SA, Crutchlow MF, Ferrari AM, Raum JC, Groff DN, Rankin MM, et al. Calcineurin signaling regulates human islet $\{$ beta\}-cell survival. $J$ Biol Chem. 2010;285(51):40050-9.

67. Kolesar JE, Wang CY, Taguchi YV, Chou SH, and Kaufman BA. Two-dimensional intact mitochondrial DNA agarose electrophoresis reveals the structural complexity of the mammalian mitochondrial genome. Nucleic Acids Res. 2013;41(4):e58.

68. Karamanlidis G, Nascimben L, Couper GS, Shekar PS, del Monte F, and Tian R. Defective DNA replication impairs mitochondrial biogenesis in human failing hearts. Circ Res. 2010;106(9):1541-8.

69. Reissaus CA, Pineros AR, Twigg AN, Orr KS, Conteh AM, Martinez MM, et al. A Versatile, Portable Intravital Microscopy Platform for Studying Beta-cell Biology In Vivo. Sci Rep. 2019;9(1):8449.

70. Iwashita H, Torii S, Nagahora N, Ishiyama M, Shioji K, Sasamoto K, et al. Live Cell Imaging of Mitochondrial Autophagy with a Novel Fluorescent Small Molecule. ACS Chem Biol. 2017;12(10):2546-51.

71. Konig J, Ott C, Hugo M, Jung T, Bulteau AL, Grune T, et al. Mitochondrial contribution to lipofuscin formation. Redox Biol. 2017;11:673-81.

72. Jayaraman S. A novel method for the detection of viable human pancreatic beta cells by flow cytometry using fluorophores that selectively detect labile zinc, mitochondrial membrane potential and protein thiols. Cytometry A. 2008;73(7):615-25. 


\section{Figures}

A)

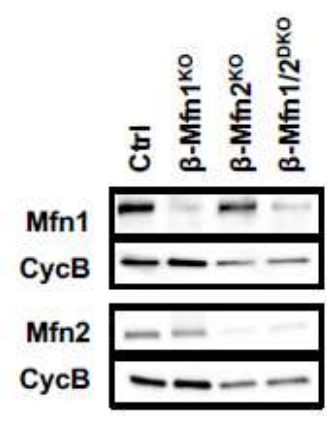

B)

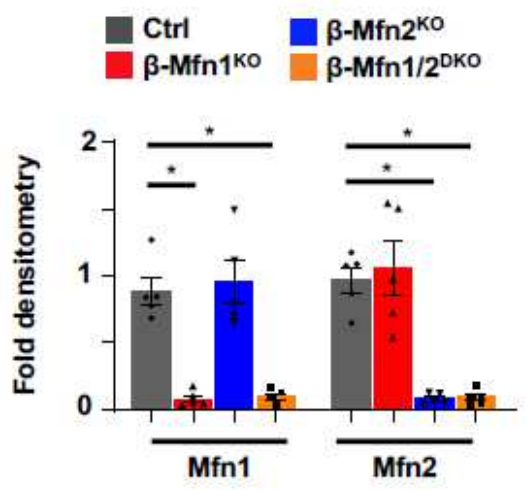

E)

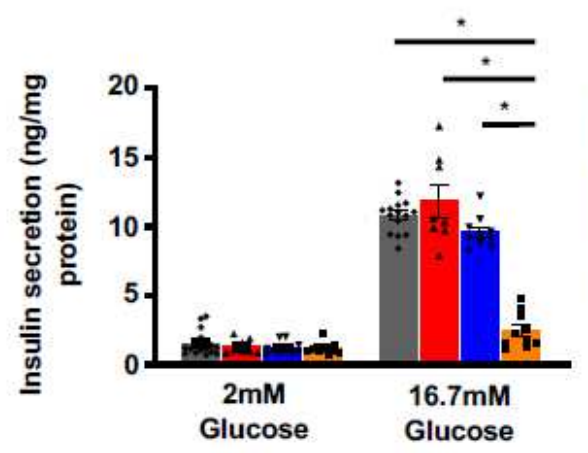

C)
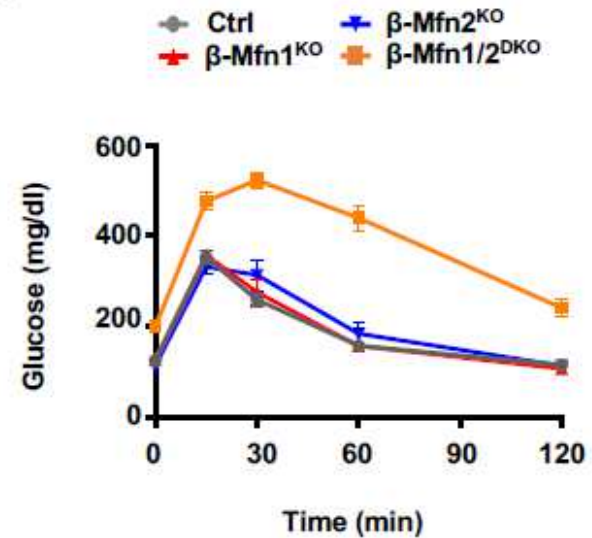

F)

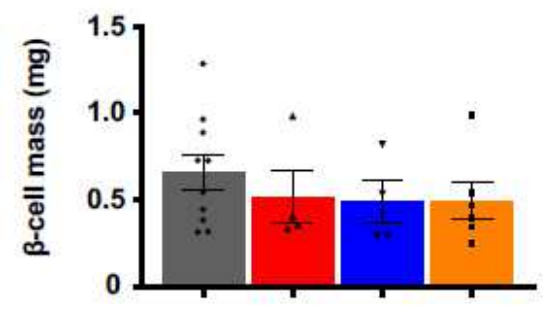

\section{Figure 1}

Loss of both Mfn1 and Mfn2 in $\beta$-cells impairs glucose tolerance and glucose-stimulated insulin release. (A) Expression of Mfn1 and Mfn2 by Western blot (WB) in islets isolated from 8-10-week-old Ctrl, $\beta$ Mfn1KO, $\beta$ - Mfn2KO and $\beta-M f n 1 / 2 D K O$ mice. Cyclophilin $B$ serves as a loading control. Representative of 4 independent mice/group. (B) Densitometry (normalized to cyclophilin B) from studies in Figure $1 \mathrm{~A}$. $n=4 /$ group; ${ }^{*}<<0.05$ by ANOVA. (C) Blood glucose concentrations measured during IPGTT of 8-week-old Ctrl, $\beta$-Mfn1KO, $\beta$-Mfn2KO and $\beta$-Mfn1/2DKO littermates. $n=6-28 /$ group. $(p<0.005$ by ANOVA for $\beta-$ Mfn1/2DKO mice vs Ctrl). (D) Serum insulin concentrations ( $n=7-24 /$ group) measured during in vivo glucose-stimulated insulin release testing in 8-week-old Ctrl, $\beta$-Mfn1KO, $\beta$-Mfn2KO and $\beta-M f n 1 / 2 D K O$ littermates. ${ }^{*} \mathrm{p}<0.05$ by ANOVA. (E) Glucose-stimulated insulin secretion following static incubations in $2 \mathrm{mM}$ and $16.7 \mathrm{mM}$ glucose, performed in isolated islets of 8-week-old Ctrl, $\beta$-Mfn1KO, $\beta-\mathrm{Mfn} 2 \mathrm{KO}$ and $\beta$ Mfn1/2DKO littermates. $n=8-16 /$ group. * $p<0.05$ by ANOVA. (F) Pancreatic $\beta$-cell mass ( $n=4-10 /$ group) measured in 10-week-old Ctrl, $\beta$-Mfn1KO, $\beta$-Mfn2KO and $\beta$-Mfn1/2DKO littermates. 


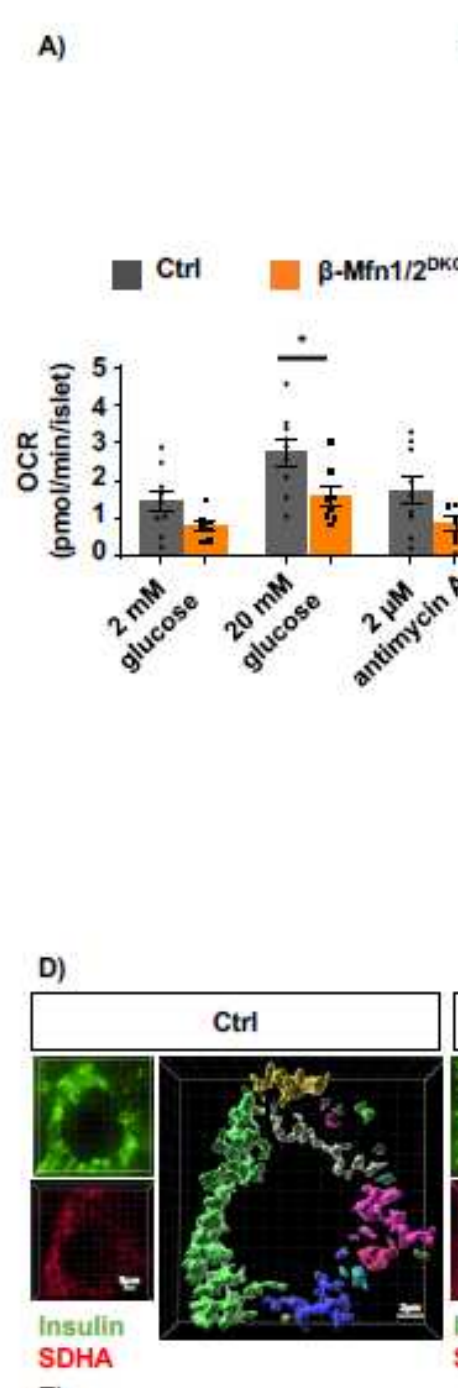

B)

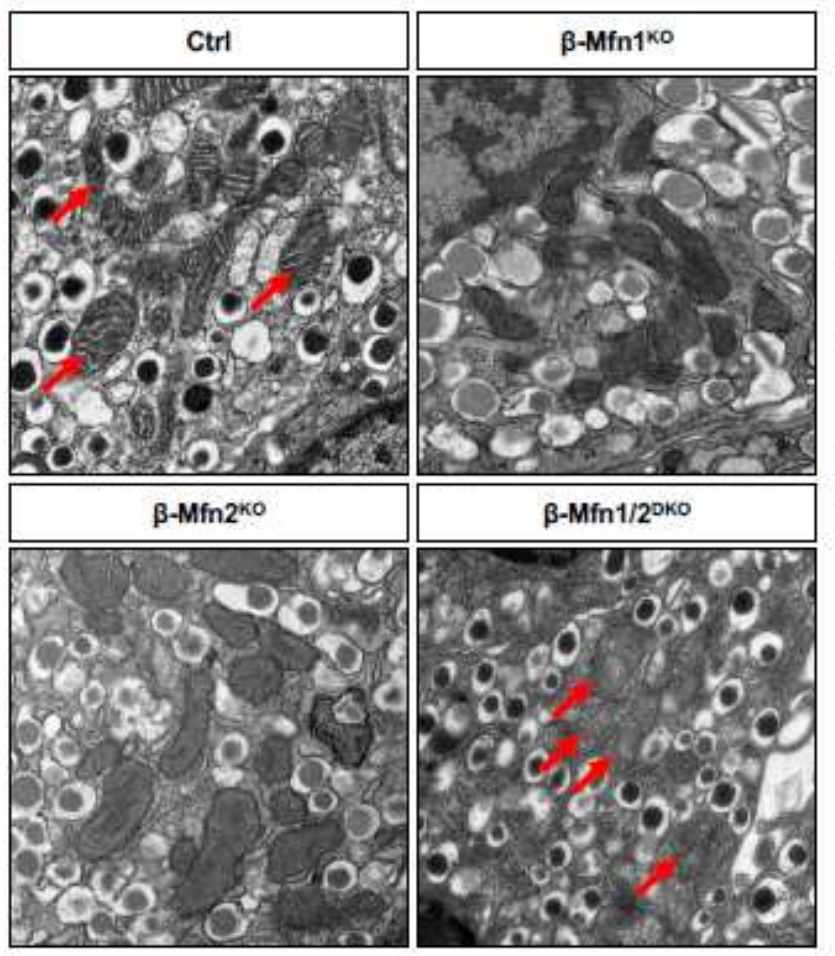

C)

단

B-Mfn1ko

$\beta-\operatorname{Mfn} 2^{\mathrm{KO}}$

$\beta-M f n 1 / 2^{\text {Dко }}$
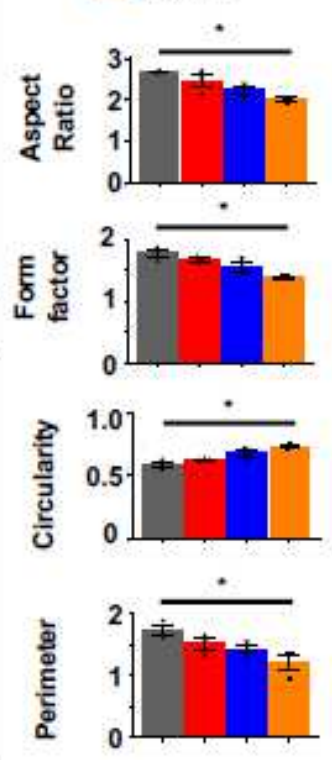

D)

E)
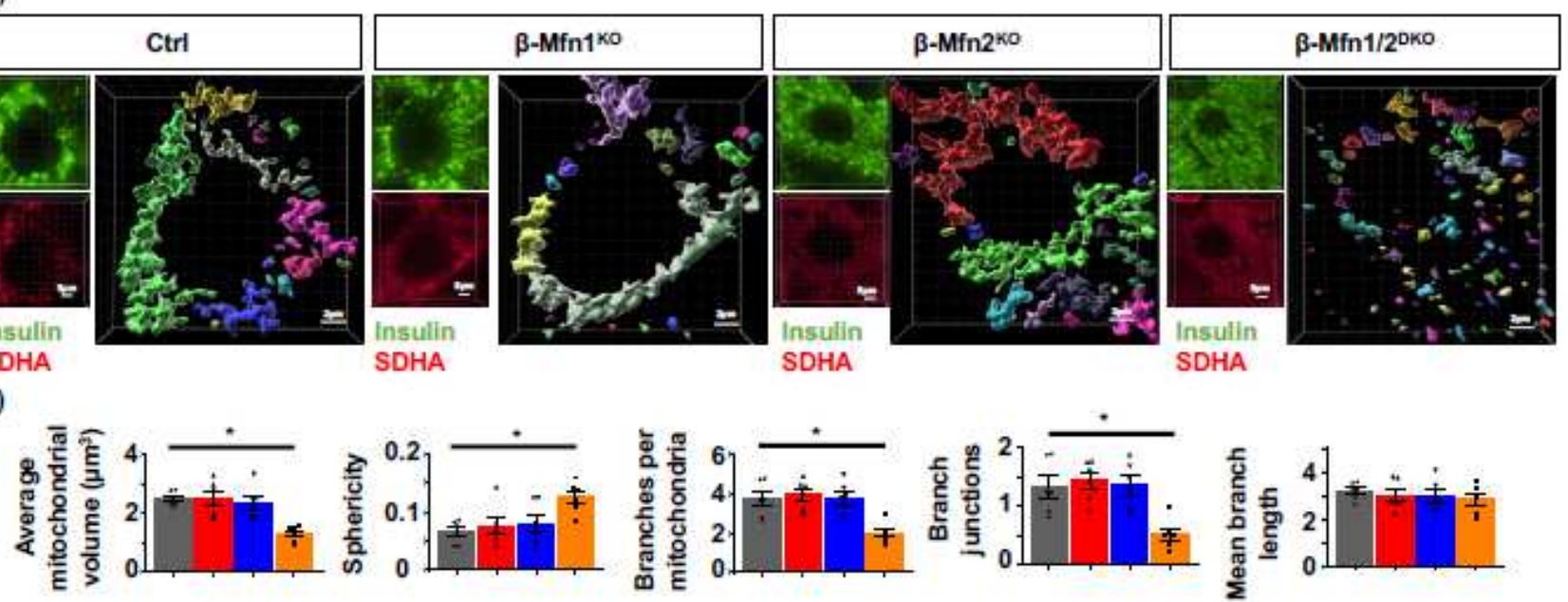

Figure 2

Combined Mfn1 and Mfn2 deficiency reduces mitochondrial respiration and leads to mitochondrial fragmentation in $\beta$-cells. (A) OCR measured following exposure to $2 \mathrm{mM}$ glucose, $20 \mathrm{mM}$ glucose, and $2 \mu \mathrm{M}$ antimycin $\mathrm{A}$ in islets isolated from 8-10-week-old Ctrl and $\beta$-Mfn1/2DKO mice by a Seahorse flux analyzer. $n=8-9 /$ group. ${ }^{*} p<0.05$ by ANOVA. (B) Representative transmission EM images of $\beta$-cells from 12week-old Ctrl, $\beta$-Mfn1KO, $\beta$-Mfn2KO and $\beta$-Mfn1/2DKO littermates. $n=3 /$ group. Mitochondria are highlighted with red arrows. (C) Quantification of mitochondrial morphology in Ctrl, $\beta$-Mfn1KO, $\beta-M f n 2 K O$ and $\beta$-Mfn1/2DKO $\beta$-cells from transmission EM images ( 125 independent mitochondria scored/animal). $n=3$ /group. ${ }^{*} p<0.05$ by ANOVA. (D) Imaris ${ }^{\circledR}$ generated threedimensional reconstruction of deconvolution immunofluorescence Z-stack images at 100X magnification stained for SDHA (see inset image - red) from pancreatic sections of Ctrl, $\beta-M f n 1 K O, \beta-M f n 2 K O$ and $\beta-M f n 1 / 2 D K O$ mice. $\beta$-cells were 
identified by insulin co-staining (inset: insulin - green). Each unique color represents a separate $\beta$ - cell mitochondrial network cluster. Representative image of 6 independent mice/group. (E) $\beta$-cell mitochondrial morphology and network analysis of deconvolution immunofluorescence Z-stack images from studies depicted in Figure 2D, stained for SDHA (and insulin) from pancreatic sections of Ctrl, $\beta$ Mfn1KO, $\beta$-Mfn2KO and $\beta$-Mfn1/2DKO mice by MitoAnalyzer. $n=6 /$ group $(\sim 150 \beta$-cells/animal were quantified). ${ }^{*} p<0.05$ by ANOVA.

A)

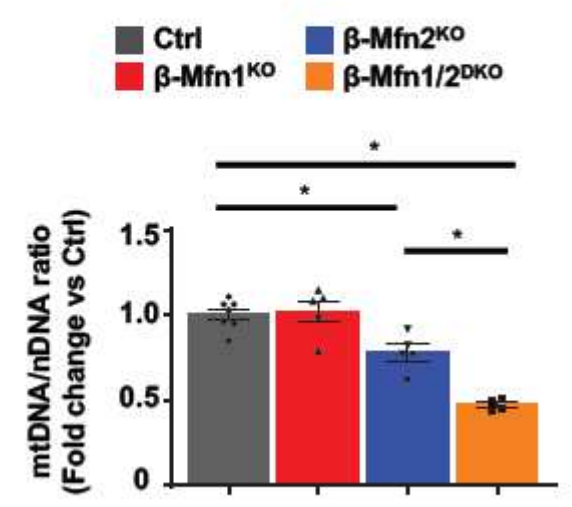

B)

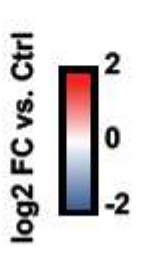

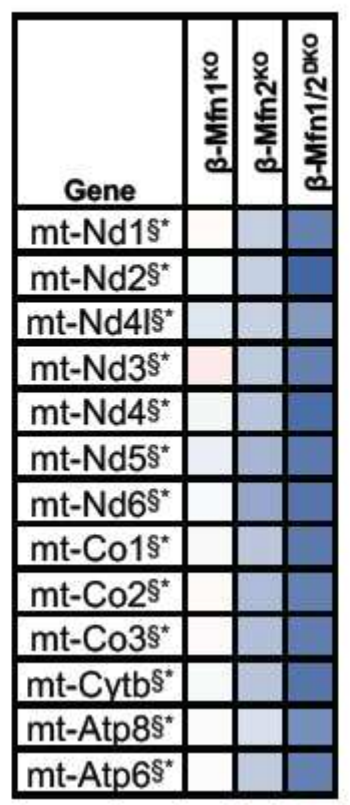

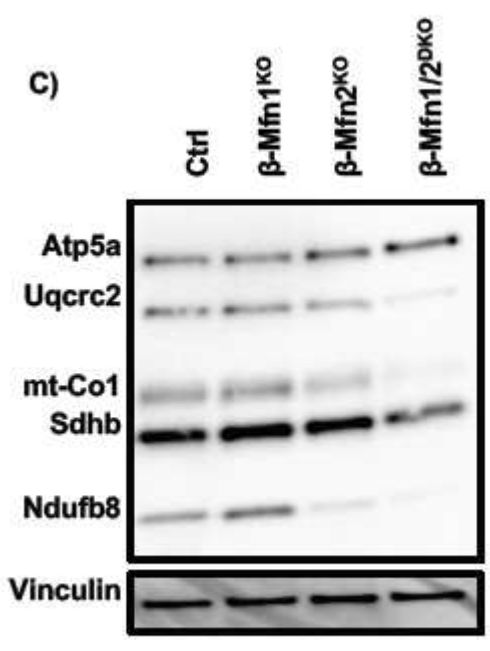

D)

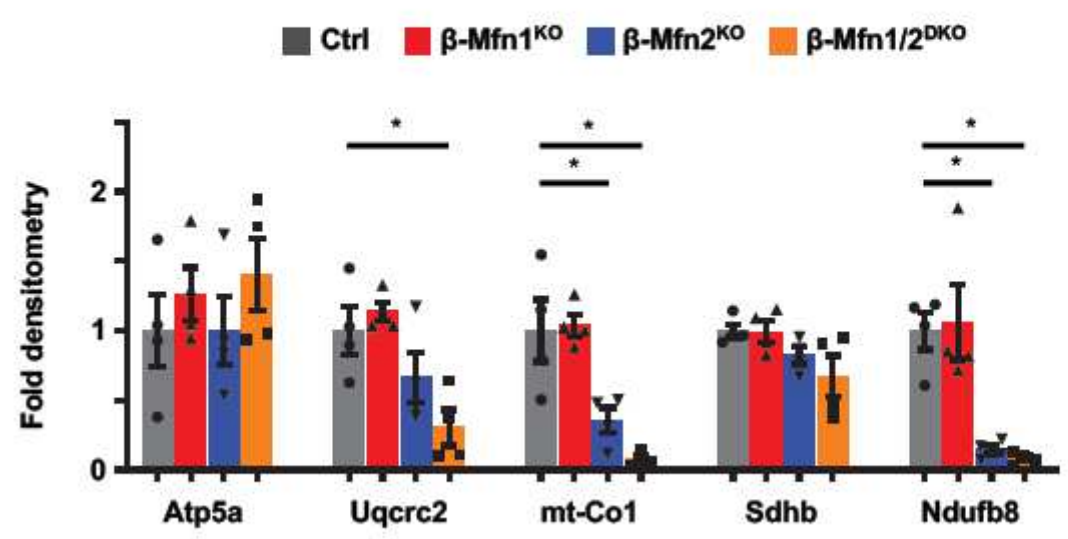

\section{Figure 3}

Mfn1/2-deficient $\beta$-cells exhibit reduced mtDNA content. (A) Relative mtDNA content measured by qPCR (normalized to nuclear DNA expression) in isolated islets of 8- week-old Ctrl, $\beta-\mathrm{Mfn} 1 \mathrm{KO}, \beta-\mathrm{Mfn} 2 \mathrm{KO}$ and $\beta$ Mfn1/2DKO littermates. $n=5-7 /$ group. ${ }^{*} p<0.05$ by ANOVA. (B) Heatmap representing relative expression (compared to littermate controls; $\mathrm{Ctrl}$ ) of mitochondrial encoded transcripts in islets isolated from $\beta$ Mfn1KO, $\beta$-Mfn2KO and $\beta$-Mfn1/2DKO mice. $n=4-6 /$ group. $\S^{*}$ Benjamini-Hochberg FDR (Padj) $<0.05$ 
comparing $\beta-\mathrm{Mfn} 2 \mathrm{KO}(\S)$ and $\beta-\mathrm{Mfn} 1 / 2 \mathrm{DKO}\left({ }^{*}\right)$ to $\mathrm{Ctrl}$. (C) Expression of OXPHOS subunit proteins by WB in islets isolated from 8-10-week-old Ctrl, $\beta-\mathrm{Mfn} 1 \mathrm{KO}, \beta-\mathrm{Mfn} 2 \mathrm{KO}$ and $\beta-\mathrm{Mfn} 1 / 2 \mathrm{DKO}$ mice. Representative of 4 independent mice/group. (D) OXPHOS subunit densitometry (normalized to Vinculin) from studies in Figure $3 \mathrm{C} . \mathrm{n}=4$ /group; ${ }^{\star} \mathrm{p}<0.05$ by ANOVA.

A)

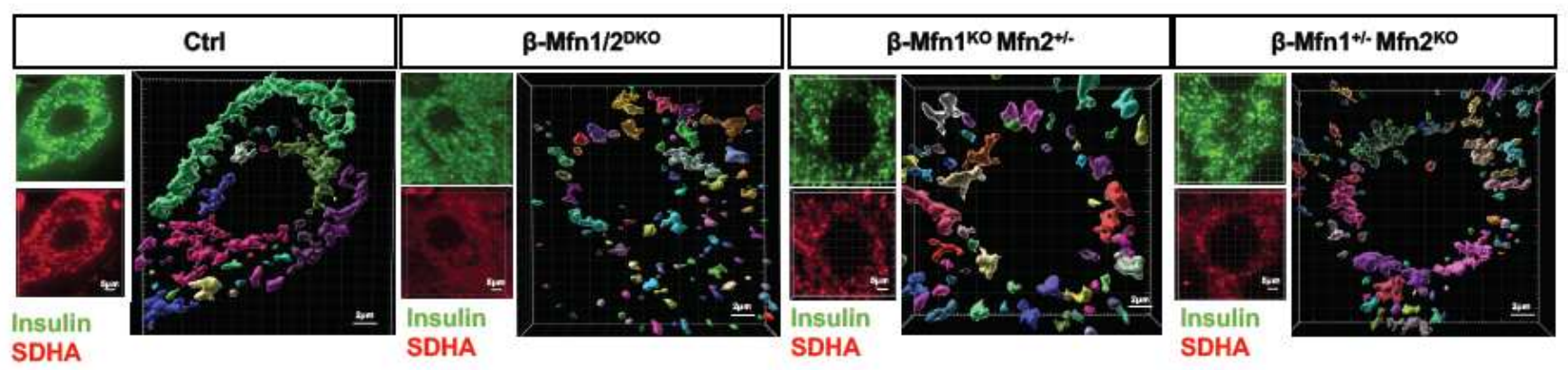

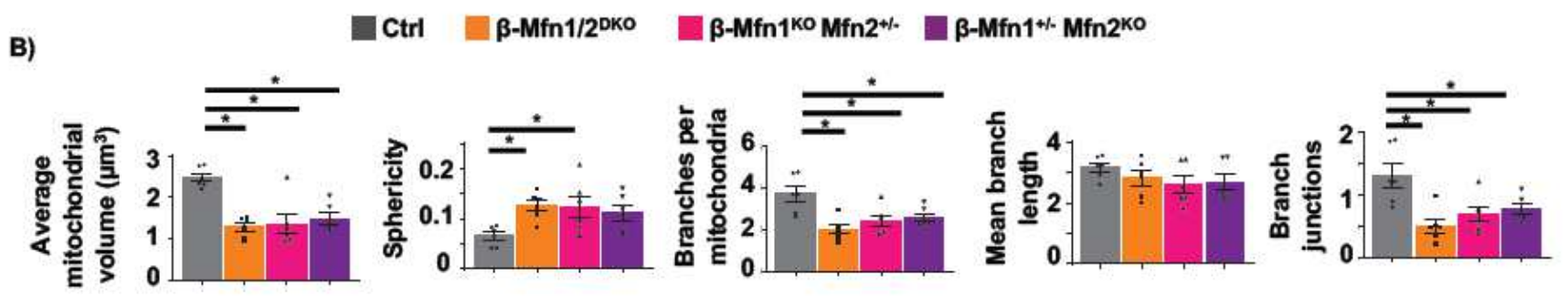

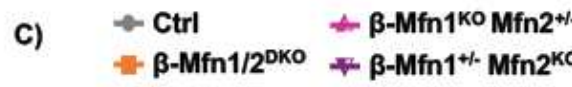

D)

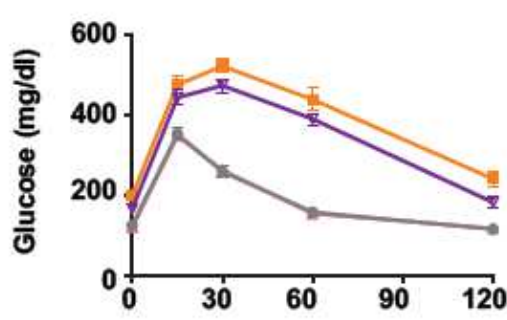

Time (min)
(1)

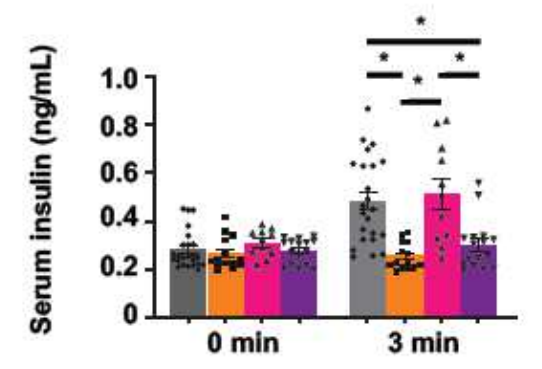

E)

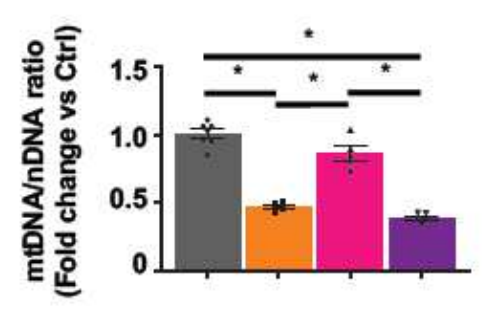

\section{Figure 4}

A single allele of $\mathrm{Mfn} 2$, but not Mfn1, maintains glycemic control by preserving $\beta$ - cell mtDNA content despite mitochondrial fragmentation. (A) Imaris $®$ generated three-dimensional reconstruction of deconvolution immunofluorescence Z-stack images at 100X magnification stained for SDHA (see inset image - red) from pancreatic sections of Ctrl, $\beta$ - Mfn1/2DKO, $\beta-M f n 1+/-M f n 2 K O$, and $\beta-M f n 1 K O M f n 2+/-$ mice. $\beta$-cells were identified by insulin co-staining (inset: insulin - green). Each unique color represents a separate $\beta$-cell mitochondrial network cluster. Representative image of 6 independent mice/group. (B) $\beta$ cell mitochondrial morphology and network analysis of deconvolution immunofluorescence Z-stack images from pancreatic sections stained for insulin and SDHA of Ctrl, $\beta$ - Mfn1/2DKO, $\beta-M f n 1+/-M f n 2 K O$, and $\beta$-Mfn1KOMfn2+/- mice by MitoAnalyzer. $n=6 /$ group (50-200 $\beta$-cells/animal were quantified). 
${ }^{*} \mathrm{p}<0.05$ by ANOVA. (C) Blood glucose concentrations measured during IPGTT of 8 -week-old Ctrl, $\beta$ Mfn1/2DKO, $\beta$-Mfn1+/-Mfn2KO, and $\beta$-Mfn1KOMfn2+/- littermates. $n=13-28 /$ group. $(p<0.05$ by ANOVA for $\beta-M f n 1+/-M f n 2 K O$ and $\beta-M f n 1 / 2 D K O$ mice vs Ctrl). (D) Serum insulin concentrations ( $n=11$ 24 /group) measured during in vivo glucose-stimulated insulin release testing in 8-week-old Ctrl, $\beta$ Mfn1/2DKO, $\beta-M f n 1+/-M f n 2 K O$, and $\beta-$ Mfn1KOMfn2+/- littermates. ${ }^{*} p<0.05$ by ANOVA. (E) Relative mtDNA content measured by qPCR (normalized to nuclear DNA expression) in isolated islets of 8-weekold Ctrl, $\beta-M f n 1 / 2 D K O, \beta-M f n 1+/-M f n 2 K O$, and $\beta$ - Mfn1KOMfn2+/- littermates. $n=5-7 /$ group. * $p<0.05$ by ANOVA. Of note, studies in Ctrl and $\beta$-Mfn1/2DKO mice were performed together alongside all $\beta$-Mfn1KO, $\beta-M f n 2 K O, \beta-M f n 1+/-M f n 2 K O$, and $\beta$-Mfn1KOMfn2+/- littermates and thus may appear twice for purposes of relevant comparisons (in Figures 1C, 1D, 2D, 2E, 3A and again in Figures 4AE).

A)

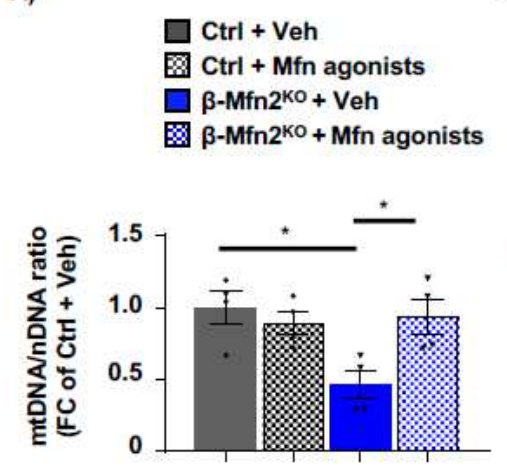

B)

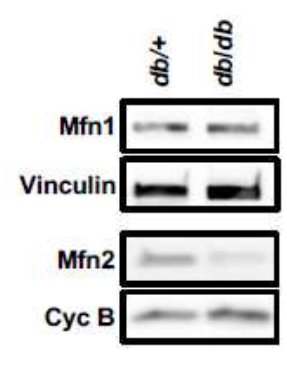

C)

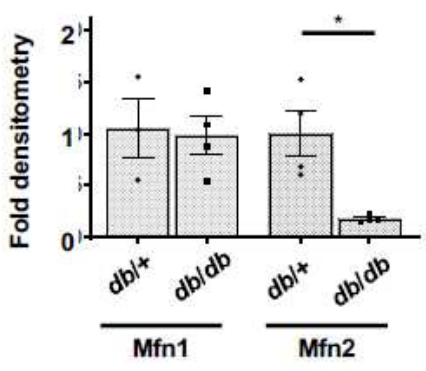

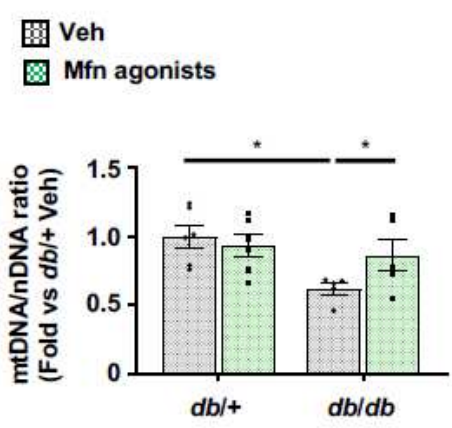

D)
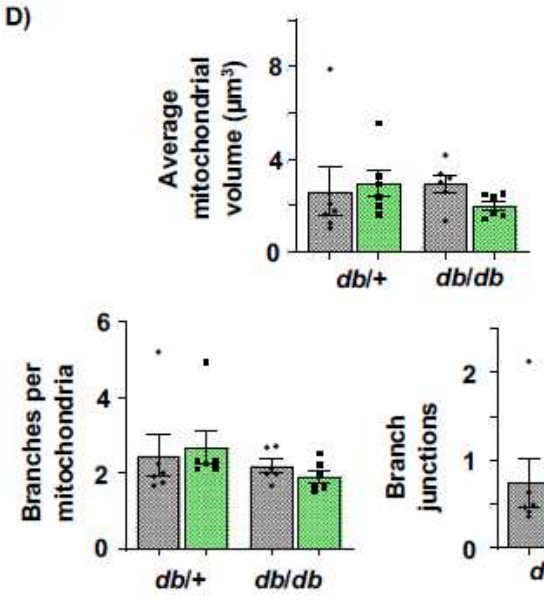
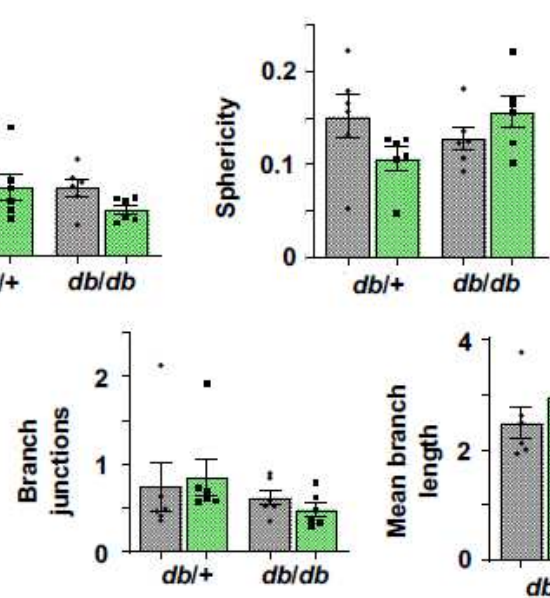

E)

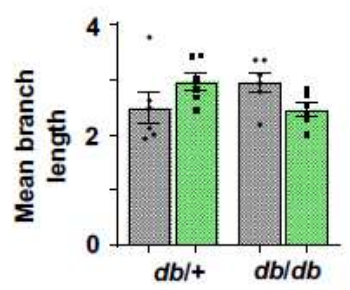

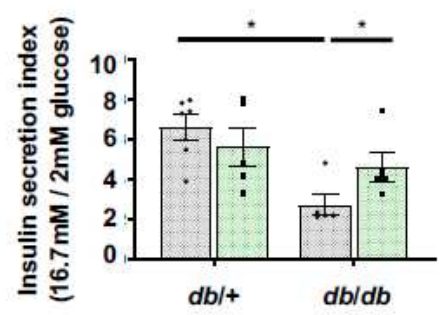

\section{Figure 5}

Mitofusin agonists restore mtDNA depletion following Mfn2 deficiency independent of changes in mitochondrial structure. (A) Relative mtDNA content measured by qPCR (normalized to nuclear DNA expression) from isolated islets of Ctrl and $\beta-M f n 2 K O$ mice following treatment with vehicle (Veh) or 0.5 $\mu \mathrm{M}$ Mfn agonists for $24 \mathrm{hrs} . \mathrm{n}=4$ /group. ${ }^{*} \mathrm{p}<0.05$ by ANOVA. (B) Expression of Mfn 1 and Mfn 2 by WB in islets isolated from 10-12-week-old db/+ and db/db mice. Vinculin and cyclophilin $B$ serve as loading 
controls. Representative image (left) of 3-4 independent mice/group. Mfn1 and 2 densitometry, normalized to vinculin or cyclophilin B (right). $n=3-4$ /group; ${ }^{*}<0.05$ by two-tailed t-test. (C) Relative mtDNA content measured by qPCR (normalized to nuclear DNA expression) from isolated islets of 10-12week-old $\mathrm{db} /+$ and $\mathrm{db} / \mathrm{db}$ mice following treatment with vehicle (Veh) or $0.5 \mu \mathrm{M}$ mitofusin agonists for 24 hrs. $n=5-6 /$ group. ${ }^{*} p<0.05$ by two-tailed $t$-test. (D) $\beta$-cell mitochondrial morphology and network analysis by MitoAnalyzer of deconvolution immunofluorescence Z-stack images, stained for SDHA (and insulin), from pancreatic islets of 10-12-week-old db/+ and db/db mice following treatment with vehicle (Veh) or $0.5 \mu \mathrm{M}$ mitofusin agonists for $24 \mathrm{hrs} . \mathrm{n}=4-6 /$ group ( 150- $200 \beta$-cells/animal were quantified). (E) Insulin secretion index from isolated islets of 10-12-week-old $\mathrm{db} /+\mathrm{and} \mathrm{db} / \mathrm{db}$ mice following treatment with vehicle (Veh) or $0.5 \mu \mathrm{M}$ mitofusin agonists for $24 \mathrm{hrs} . \mathrm{n}=5-6 /$ group. ${ }^{*} \mathrm{p}<0.05$ by two-tailed t-test. 


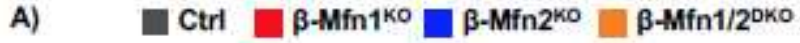

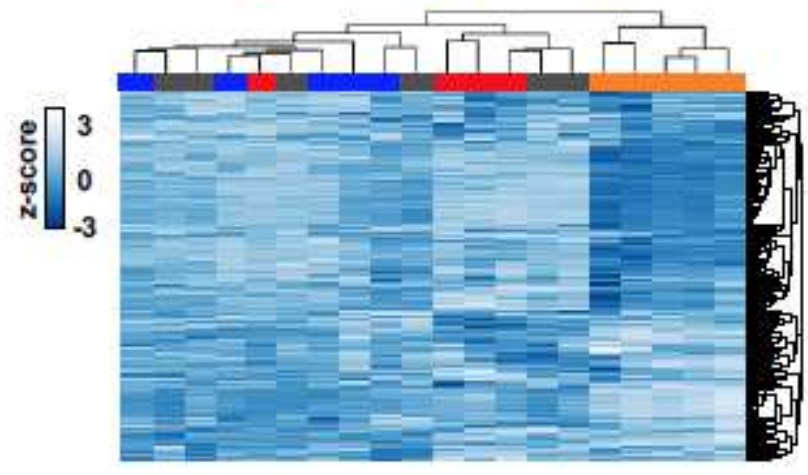

B)

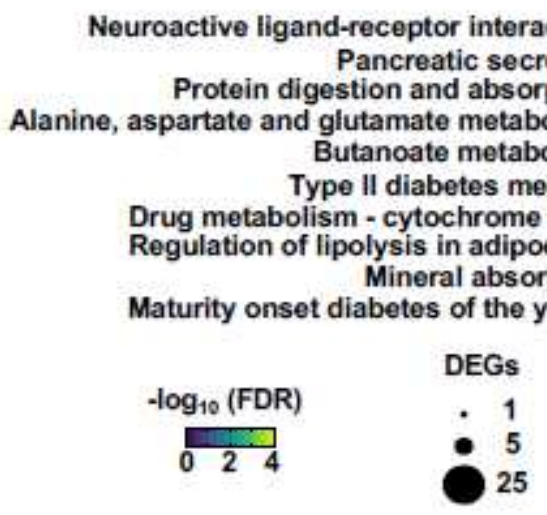

C)

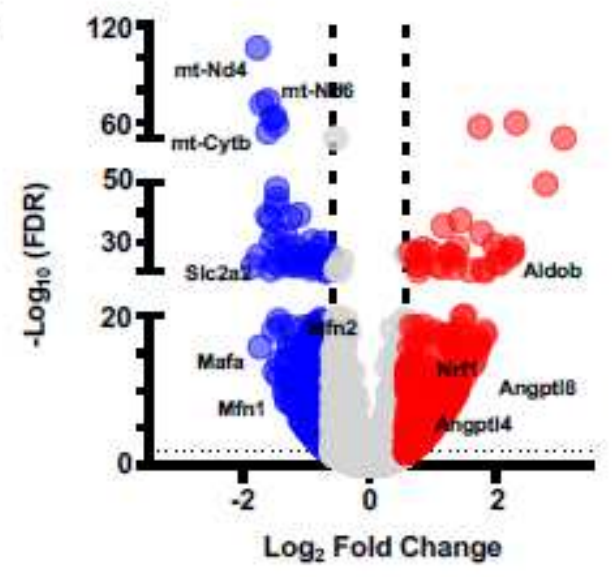

D)

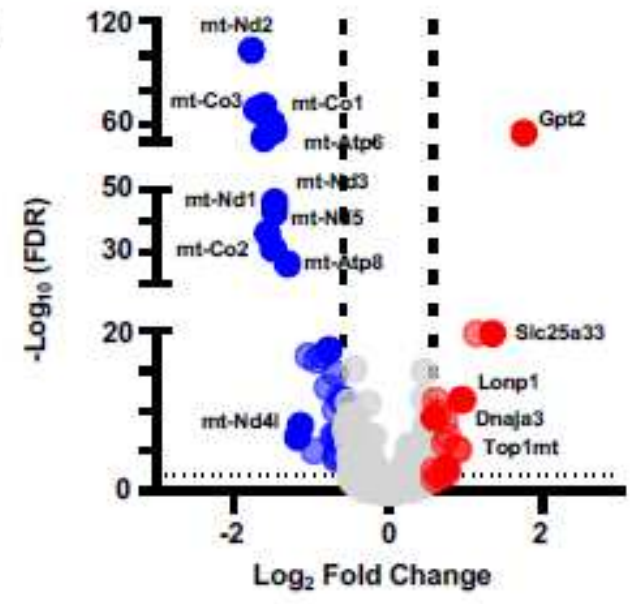

E)

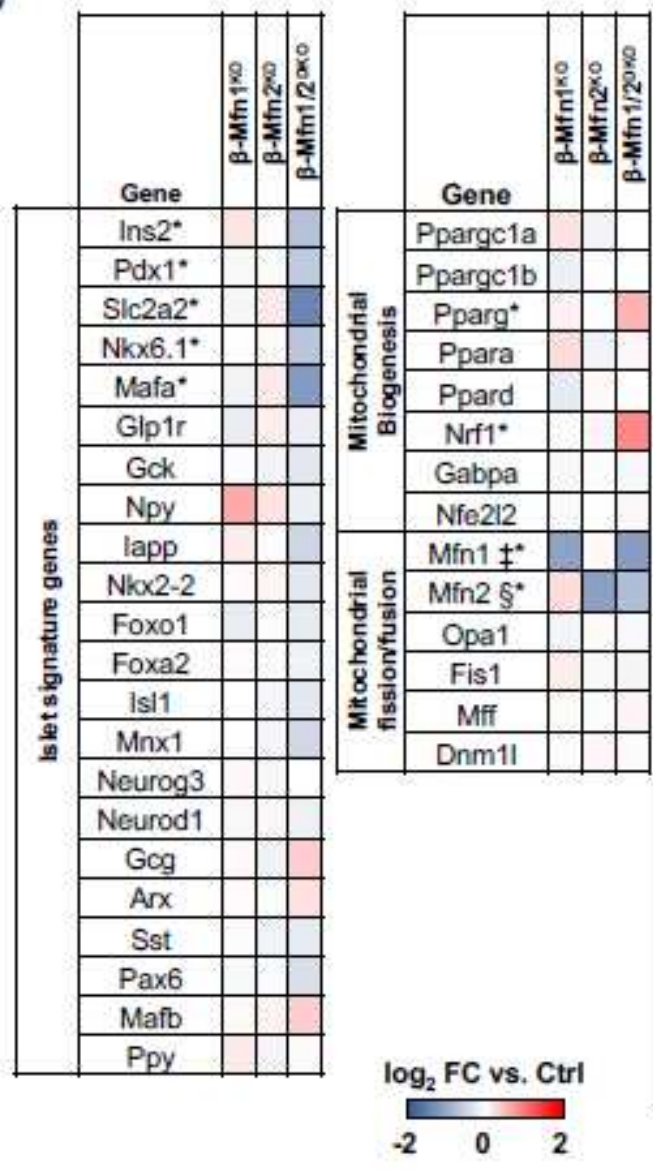

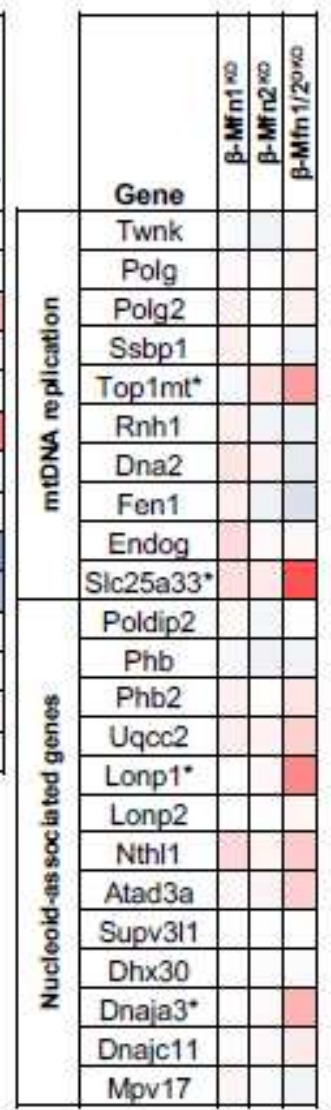

Figure 6

Mfn1/2-deficiency induces expression of genes associated with mtDNA replication. (A) Hierarchical clustering of RNAseq samples displaying the 500 genes with the highest mean expression in islets of 12week-old mice. $n=4-6 /$ group. (B) Pathway analysis of top differentially regulated pathways in $\beta$ Mfn1/2DKO islets compared to littermate Ctrl islets, demarcated by both false discovery rate (FDR) and the number of significantly differentially expressed genes (DEGs) per pathway. $n=4-6 /$ group. (C) Volcano plot depicting differential RNA expression in islets of $\beta-M f n 1 / 2 D K O$ mice compared to littermate Ctrl 
mice. Significantly differentially expressed genes demarcated by $\log 10 \mathrm{FDR}>$ or $<2$ and $\log 2$ fold change (FC) $>$ or $<0.6$. $n=4-6 /$ group. (D) Volcano plot depicting differential RNA expression of MitoCarta 2.0 targets in islets of $\beta-\mathrm{Mfn} 1 / 2 \mathrm{DKO}$ mice compared to littermate $\mathrm{Ctrl}$ mice. Significantly differentially expressed genes demarcated by -log10 FDR $>$ or $<2$ and log2 fold change $(F C)>$ or $<0.6$. $n=4-6 /$ group. (E) Differential RNA expression heatmap of selected genes from islets of $\beta$-Mfn1KO, $\beta$-Mfn2KO and $\beta$ Mfn1/2DKO mice compared to littermate Ctrl mice. up. $\ddagger \S^{\star}$ Benjamini-Hochberg FDR (Padj) $<0.05$ comparing $\beta-M f n 1 K O(\ddagger), \beta-M f n 2 K O(\S)$ and $\beta-M f n 1 / 2 D K O(*)$ to $\mathrm{Ctrl} . n=4-6 /$ group.

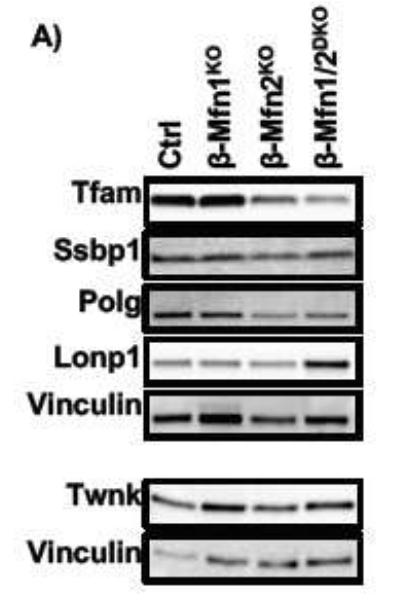

D)

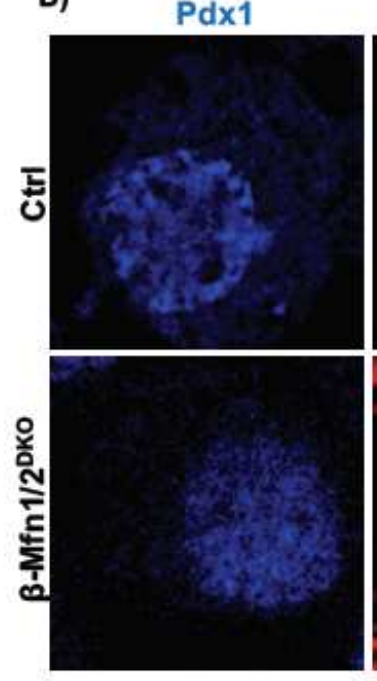

G)

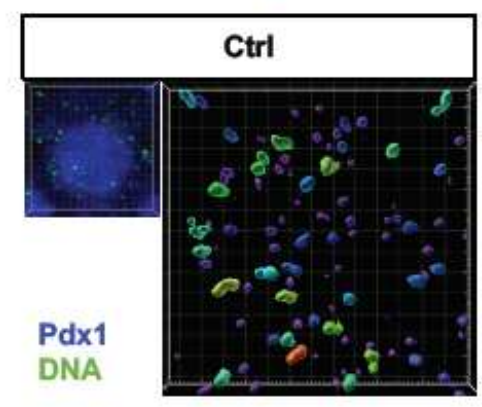

B)

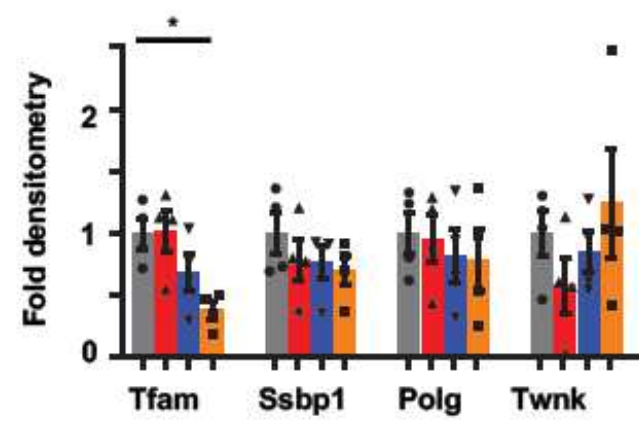

Ssbp1
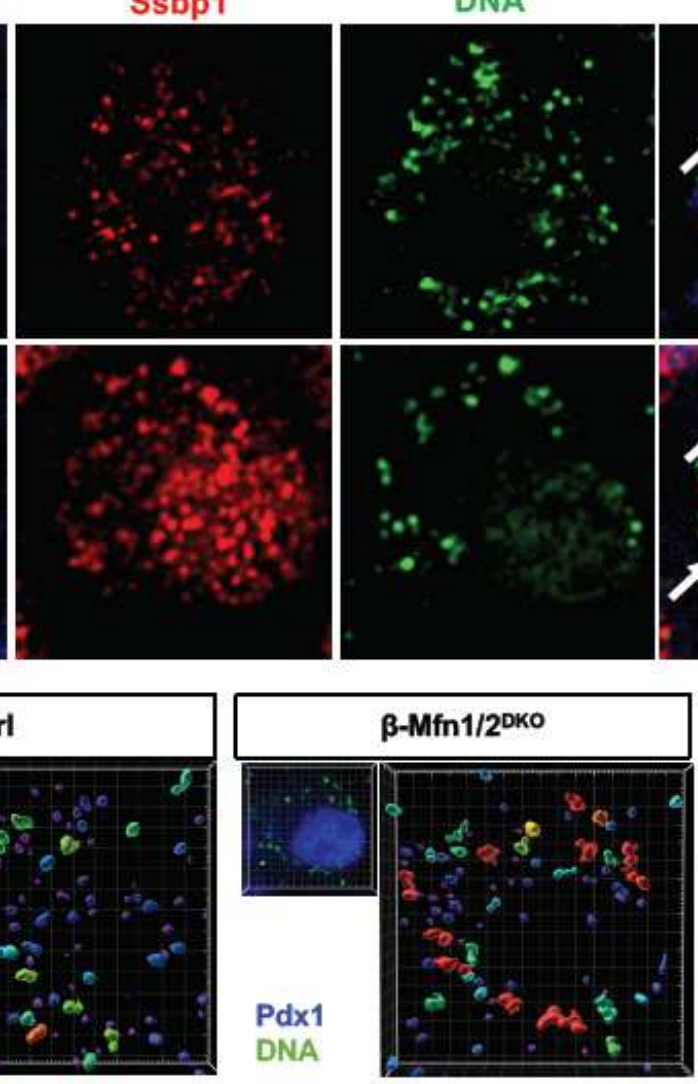

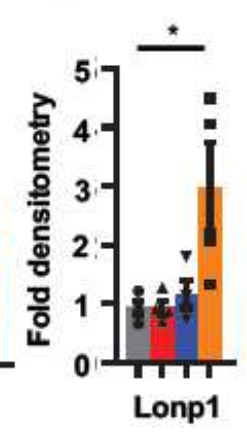

Merge

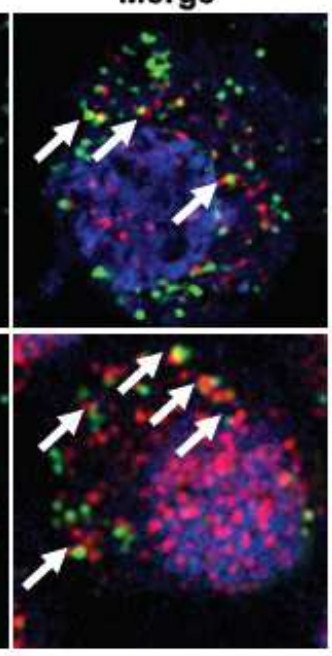

H)

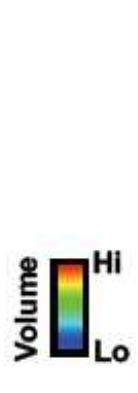

C)

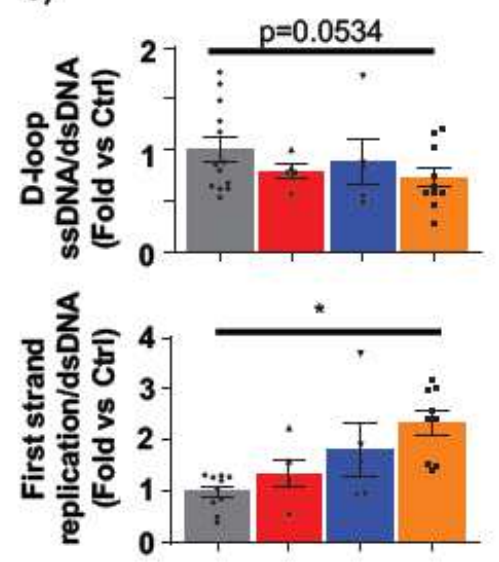

E)
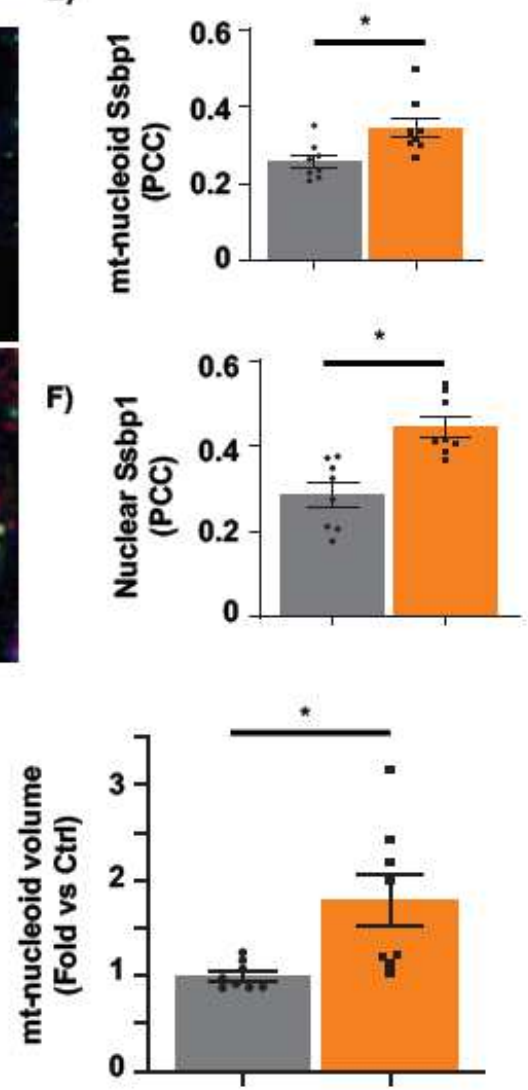

Figure 7

Mfn1 and 2 maintain expression of Tfam in $\beta$-cells. (A) Expression of Tfam, key replisome proteins, and LonP1 by Western blot (WB) in islets isolated from 11- week-old Ctrl, $\beta$-Mfn1KO, $\beta$-Mfn2KO and $\beta$ - 
Mfn1/2DKO mice. Representative of 4 independent mice per group. (B) Densitometry (normalized to Vinculin) from studies in Figure 7A. $n=4 /$ group; ${ }^{*}<0.05$ by ANOVA. (C) Quantification of single strand mtDNA products measured by qPCR in isolated islets from 8-weekold Ctrl, $\beta$-Mfn1 KO, $\beta$-Mfn2KO and $\beta$ Mfn1/2DKO littermates. $n=5-13 /$ group. ${ }^{*} p<0.05$ by ANOVA. (D) Deconvolution immunofluorescence image at 100X magnification of islets from Ctrl and $\beta-M f n 1 / 2 D K O$ mice stained for Ssbp1 (red), mtDNA (green), and Pdx1 (blue). Representative of 8 independent experiments. White arrows demarcate colocalized Ssbp1+ mtDNA+ structures. (E) Quantification of mitochondrial nucleoid Ssbp1 localization (Ssbp1+ mtDNA+ co-localization) in Ctrl and $\beta$-Mfn1/2DKO $\beta$-cells from studies depicted in Figure 7D by Pearson's correlation coefficient (PCC). $n=8$ /group. ${ }^{*} p<0.05$ by two-tailed t-test. ( $100 \beta$-cells from each animal per group were analyzed). (F) Quantification of nuclear Ssbp1 localization in Ctrl and $\beta$ Mfn1/2DKO $\beta$-cells from studies depicted in Figure 7D by Pearson's correlation coefficient (PCC). $\mathrm{n}=8$ /group. ${ }^{*} \mathrm{p}<0.05$ by two-tailed $\mathrm{t}$-test. ( $100 \beta$-cells from each animal per group were analyzed). $(\mathrm{G})$ Imaris ${ }^{\circledR}$ generated 3D reconstruction of deconvolution immunofluorescence Z-stack images at $100 \mathrm{X}$ magnification stained for DNA of $\beta$-cells (see inset image - Pdx 1 , blue, DNA, green) from Ctrl and $\beta$ Mfn1/2DKO mice. Colors represent relative mitochondrial volume. Representative image of 8 independent mice/group. (H) Quantification of relative nucleoid volume of Ctrl and $\beta$-Mfn1/2DKO $\beta$-cells from studies depicted in Figure 7G. $n=8$ /group. ${ }^{\star} p<0.05$ by two-tailed t-test. $(\sim 100 \beta$-cells from each animal per group were analyzed). 


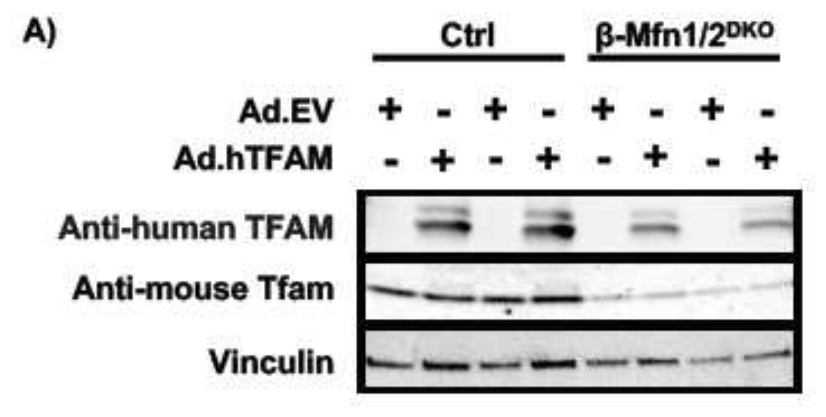

C)

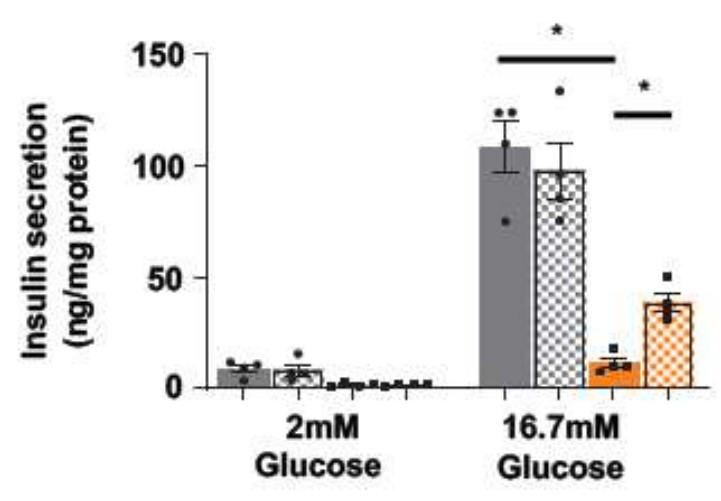

B)

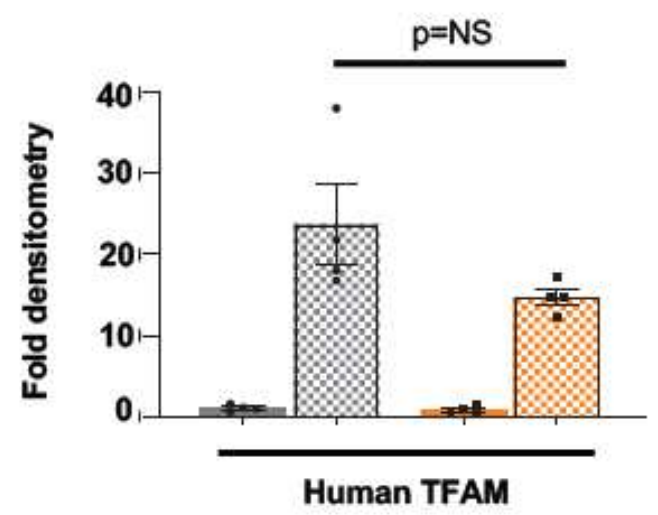

D)

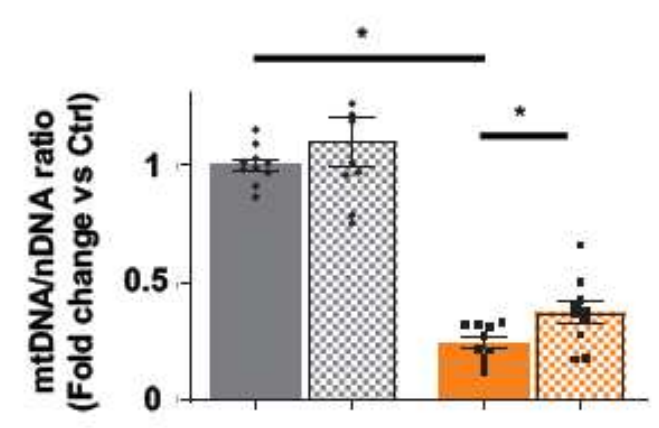

Figure 8

Mfn1 and 2 maintain $\beta$-cell function through Tfam-mediated mtDNA copy number control. (A) Expression of mouse-specific Tfam and human-specific TFAM by WB in islets isolated from 10-15-week-old Ctrl and $\beta$-Mfn1/2DKO mice, transduced with empty vector control (Ad.EV) or human TFAM-overexpressing (Ad.hTFAM) adenoviral particles. Representative of 4 independent mice/group. (B) Densitometry (normalized to cyclophilin B) from studies in Figure 8A. n=4/group; $p=N S$ (non-significant) by t-test. (C) Glucose-stimulated insulin secretion following static incubations in $2 \mathrm{mM}$ and $16.7 \mathrm{mM}$ glucose, performed in isolated Ctrl and $\beta$ - Mfn1/2DKO islets following transduction with Ad.EV or Ad.hTFAM adenoviral particles. $n=4$ /group. ${ }^{*} p<0.05$ by ANOVA. (D) Relative mtDNA content measured by $q P C R$ (normalized to nuclear DNA expression) from isolated Ctrl and $\beta$-Mfn1/2DKO islets following transduction with Ad.EV or Ad.hTFAM adenoviral particles. $n=8-10$ /group. ${ }^{*} p<0.05$ by ANOVA.

\section{Supplementary Files}

This is a list of supplementary files associated with this preprint. Click to download. 
- SidaralaSupplementarytable1.pdf

- MfnManuscriptSupplementaryFigures03082021.pdf 